\title{
Effect of the isotope mass on the turbulent transport at the edge of L-mode plasmas in ASDEX Upgrade and JET-ILW
}

\author{
N. Bonanomi ${ }^{1}$, C. Angioni ${ }^{1}$, P. C. Crandall ${ }^{1,3}$, A. Di Siena ${ }^{1}$, C. F. Maggi ${ }^{2}$, P. A. Schneider ${ }^{1}$ \\ the ASDEX Upgrade Team, the EUROFusion MST1 Team* and JET Contributors**
}

1) Max Planck Institute for Plasma Physics, Boltzmannstr. 2, 85748 Garching, Germany

2) Culham Centre for Fusion Energy, Abingdon, OX14 3DB, UK

3) University of California, Los Angeles, USA

*See the author list H. Meyer et al 2017 Nucl. Fusion 57102014

\begin{abstract}
**See the author list of "Overview of the JET preparation for Deuterium-Tritium Operation" by E. Joffrin et al. to be published in Nuclear Fusion Special issue: overview and summary reports from the 27th Fusion Energy Conference (Ahmedabad, India, 22-27 October 2018)

Abstract

The nature of the turbulent transport in the edge region of ASDEX Upgrade and JET-ILW deuterium $(D)$ and hydrogen $(H)$ L-mode plasmas is studied using local gyro-kinetic simulations. For both devices the turbulence is found to be dominated by an electron drift-wave destabilized by the high collisionality in the plasma edge and strongly related to the kinetic electron dynamics. The isotope ion mass is found to have an important impact on the edge turbulence. In linear simulations wider eigenfunctions of the electrostatic potential with lower isotope mass translate in a lower threshold in the normalized electron temperature gradient and in a higher linear growth rate. In nonlinear simulations higher turbulent transport is predicted with lower isotope mass. The effect of the isotope mass on the turbulent fluxes is enhanced by electromagnetic effects, which are found to be important in non-linear simulations. Remarkably, when considering all the effects in the non-linear simulations and the experimental uncertainties, the predicted fluxes can reproduce the experimental fluxes and their behavior with the isotope mass.
\end{abstract}

\section{Introduction}

Since the first comparisons between different hydrogen isotope plasmas in tokamaks it appeared that deuterium (D) plasmas have a better performance than hydrogen $(\mathrm{H})$ plasmas $[1,2,3]$. Experiments with tritium (T) also confirm this trend with the isotope mass [4]. These observations are in opposition to early theoretical predictions that indicate a gyro-Bohm mass scaling for the turbulent transport $\left(\chi_{i} \propto \sqrt{m_{i}}\right.$, see e.g. Ref.[5]), this contrast between theory and experiment generally referred to as 'isotope effect'. This discrepancy has been the focus of a series of recent studies on the effect of the isotope mass on the turbulent transport that identified various mechanisms that can break the gyro-Bohm mass expectations. Kinetic electrons [6], electromagnetic effects $[7,8]$, collisions [9, 10], differences in the zonal flows [11, 12, 13], differences in the critical gradient threshold, $E \times B$ flow shear $[7,14]$ and differences in sources and sinks have all been found able to cause deviations from 
the gyro-Bohm scaling with the isotope mass. Also global effects, known to being able to break the gyro-Bohm scaling [15], could depend on the isotope mass. One important aspect for the comprehension of the isotope effect is the role of the isotope mass at the plasma edge. A general experimental observation is that the effects of the isotope mass in the plasma edge of L-modes and the plasma pedestal of H-modes play an important role. The L-H power threshold $[1,3,16,17]$ and the $\mathrm{H}$-mode pedestal $[1,3,18,19]$, essential parameters for the next generation of tokamak reactors, have been all found to depend on the ion isotope mass. In particular, more heating power and/or gas puff are needed in general in $H$ plasmas in order to obtain similar edge and pedestal pressure profiles of $D$ plasmas. Understanding how the isotope ion mass acts in the plasma edge region is then essential for predicting future reactors such as ITER (International Thermonuclear Experimental Reactor). It is still uncertain if the first phase of operations of ITER will be in $H$ or $H e$ plasmas [20], this uncertainty still open due to our insufficiently precise extrapolation to ITER from present results. It will also be important to be able to predict ITER nuclear operations in $D$ and $D-T$ using the data from the the first non-nuclear phase. A clear understanding of the nature of the turbulent transport in the edge and of its relation with the isotope mass is then highly desirable. Past studies have already focused on tokamak edge and pedestal turbulence suggesting several instabilities to explain the turbulent transport in this region. Trapped Electron Modes (TEM) [21], Ion Temperature Gradient (ITG) modes [21], Electron Temperature Gradient (ETG) modes [21, 22, 23], Resistive Ballooning Modes (RBM) [24, 25, 26, 27], Kinetic Ballooning Modes [21], Micro Tearing Modes [21, 22, 23] and electron drift-wave modes destabilized by collisions [6, 28, 29, 24, 30, 25] have all been proposed to explain the edge and pedestal turbulent transport. Especially in Ref.[29, 24, 30], the nature of the turbulence in the edge tokamak region with L-mode relevant parameters was studied in detail, concluding that drift-wave instabilities play a major role and that electromagnetic effects are fundamental. The effect of the isotope mass on the turbulence was discussed in Ref.[28], where a higher non-adiabatic response of electrons with lower isotope mass was hypothesized for certain plasma conditions and a collisional parameter, $C \propto m_{i}^{-1}$ [28, 24], was proposed for the identification of the relevant plasma conditions for this effect to be important. Other important parameters has been identified in Ref. $[24,29,30,31]$ and are $C=\left(0.51 \nu_{e i} \frac{L_{\perp}}{c_{s}} \frac{m_{e}}{m_{i}}\left(\frac{q R}{L_{\perp}}\right)^{2}\right), \hat{\mu}=\frac{m_{e}}{M_{i}}\left(\frac{q R}{L_{\perp}}\right)^{2}$

and $\nu_{B}=C \cdot \omega_{B}=\left(0.51 \nu_{e i} \frac{L_{\perp}}{c_{s}} \frac{m_{e}}{m_{i}}\left(\frac{q R}{L_{\perp}}\right)^{2}\right) \cdot\left(\frac{2 L_{\perp}}{R}\right), q$ being the safety factor and $L_{\perp}$ the characteristic length of the steepest edge profile. Values of $\hat{\mu}, C, \hat{\beta} \gtrsim 1$ indicate that non-adiabatic electron response due to inertia, collisional effects and electromagnetic effects are important for the turbulence. Furthermore, a limit between drift-wave and interchange turbulence can be identified for $\nu_{B} \geq 1$ [24]. These parameters have been found to be precious guides for our study and a list of their values in the discharges studies in this work can be found in Table 1 .

With these past theoretical and numerical results in mind, in this paper we study the nature of the turbulent transport in ASDEX Upgrade and JET-ILW (Joint European Torus with the ITER-Like Wall) L-modes edge focusing on the effect of the isotope mass on the turbulence. We use gyro-kinetic simulations with experimental input parameters and compare the results with the available experimental measurements. Doing so, we can study and compare the nature of the turbulent flux in experimental relevant conditions in different machines. The paper is organized as follows: in section 2 the experimental data used in this work are briefly described and in section 3 the numerical simulation set-up is presented. In section 4 the results from the gyro-kinetic simulations are described while the conclusions are reported in section 5 .

\section{Experimental data}

The study presented in this paper is based on the data from the ASDEX Upgrade tokamak (major radius $R_{0}=1.65 \mathrm{~m}$, minor radius $a=0.5 \mathrm{~m}$ ) with a full tungsten wall and from the JET tokamak (major radius 
$R_{0}=2.96 \mathrm{~m}$, minor radius $a=1 \mathrm{~m}$ ) with the ITER-like wall, i.e. with a tungsten divertor and a beryllium main chamber. For both devices a pair of hydrogen $(H)$ and deuterium $(D)$ L-mode discharges with same toroidal field, plasma current, plasma shape and with matched temperature and density profiles have been considered. In particular $B_{T}=2.5 \mathrm{~T}$ for ASDEX Upgrade and $B_{T}=3.0 \mathrm{~T}$ for JET while $I_{p}=0.8 \mathrm{MA}$ for ASDEX Upgrade and $I_{p}=2.5 \mathrm{MA}$ for JET. In order to obtain similar temperature profiles, $\sim 30 \%$ more heating power in ASDEX Upgrade and $\sim 40 \%$ more heating power in JET were necessary in the $H$ discharges, while, to match the density profiles, $0-30 \%$ more gas puff in ASDEX Upgrade and $\sim 30 \%$ more gas puff in JET were necessary in $H$ discharges. One difference between the studied JET and ASDEX Upgrade discharges is the main heating power. ECRH (Electron Cyclotron Resonance Heating) heating was used in ASDEX Upgrade (0.5 $M W$ in D and $0.81 M W$ in $\mathrm{H}$ ), while NBI (Neutral Beam Injection) heating was used in JET (3.4 $M W$ in D and $4.5 M W$ in $\mathrm{H}$ ). More information on the experimental settings and methods used for analyzing these discharges can be found in Ref.[3] for JET and in Ref.[32] for ASDEX Upgrade. The fits of the plasma edge profiles of the plasmas studied in this paper are shown in Fig.1. While in both cases the density and the ion and electron temperatures are almost matched, as well as the values of $R / L_{T i, e}=-R \cdot \nabla T_{i, e} / T_{i, e}$, the normalized density gradient $R / L_{n}=-R \cdot \nabla n / n$ is lower in the $H$ plasmas, especially in ASDEX Upgrade. This difference in the edge density peaking between $H$ and $D$ plasmas has been already observed in the past in ASDEX Upgrade and could play an important role in the different $\mathrm{L}-\mathrm{H}$ threshold between $\mathrm{H}$ and $\mathrm{D}$ plasmas. It is important to note that interpretative simulations of the the JET discharges have been performed using the EDGE2D/EIRENE code and are reported in Ref.[3]. The simulations predict higher particle and heat diffusivity coefficients for hydrogen in the edge and SOL (Scrape Off Layer) region, pointing to a major role of the edge transport in explaining the differences between these $H$ and $D$ plasmas. These results also suggest that the different neutral penetrations of $H$ and $D$ neutrals and their influence on the particle source profiles cannot explain the difference observed in the density gradients.

\section{Gyro-kinetic simulation set-up}

Using the experimental parameters of the discharges presented in section 2 as input, linear and non-linear gyro-kinetic simulations with the GENE (Gyrokinetic Electromagnetic Numerical Experiment) code [33, 34] have been performed in order to study the micro-instabilities in the plasma edge. GENE solves the gyrokinetic Vlasov equations $[35,36]$ coupled with the Maxwell equations within a $\delta f$ approximation and using a set of field aligned coordinates $\left\{x, y, z, v_{\|}, \mu\right\} . z$ is the coordinate along the magnetic field line, $x$ is the radial coordinate, $y$ is the binormal coordinate, $v_{\|}$is the parallel velocity and $\mu$ is the magnetic momentum. The simulations are carried out using realistic geometry (reconstructed from numerical equilibrium files provided by equilibrium solvers), collisions, finite- $\beta$ effects (considering both $B_{\perp}$ and $B_{\|}$fluctuations), kinetic ions and kinetic electrons with the realistic mass ratio. The external flow shear $\gamma_{E x B}$ has not been considered in the simulations but its effect has been tested for one ASDEX Upgrade case. No impurities have been considered in the simulations, the experimental values of $Z_{\text {eff }}$ of these discharges being low ( $Z_{\text {eff }} \approx 1.2-1.3$ ), as is typical for metal machines. Typical grid parameters in the non-linear simulations were as follows: perpendicular box sizes $\left[L_{x}, L_{y}\right] \approx[500,250] \rho_{s}$, phase-space grid discretization $\left[n_{x}, n_{y}, n_{z}, n_{v \|}, n_{\mu}\right] \approx[512,62-72,70,32,16]$ and $0.02 \lesssim k_{y} \rho_{s} \lesssim 1.4$.

All the simulations in this work have been done in the local limit except few linear global simulations. This choice has been dictated by the fact that the present global electromagnetic version of the code is unstable when the magnetic field fluctuations play an important role (as it is in present cases). The use of periodic boundary conditions in local simulations had to deal with the appearance of radially elongated structures of the potentials, found to be much larger than what is usually found in a core simulation. In particular the large $L_{x}$ box size was necessary in the electromagnetic simulations due to the appearance of radially elongated structures of the 
parallel magnetic potential $A_{\|}$(shown in Fig.2) that could not be resolved with a radial box size $L_{x}<400 \rho_{s}$. The electrostatic potential $(\phi)$ structures, that are also quite large compared to usual core simulations, could be resolved with narrower box $\left(L_{x} \approx 120\right)$. Another way to avoid $A_{\|}$streamers would have been to suppress the potentials at the box radial boundaries using Dirichlet radial boundary conditions. This way has not been tested in this work although it has been used in past local gyrokinetic study of the H-mode pedestal [21, 23] and in past gyro-fluid and gyro-kinetic simulations of the L-mode edge [24, 29, 30]. As will be shown in section 4, our simulations confirm many of the properties of the edge turbulence found in Ref. [24, 29, 30]. This, with the facts that the nonlinear fluxes are related to $\phi$ fluctuations and not to $A_{\|}$fluctuations and that the results have not been found to depend on the radial box size once the electrostatic potential structures are well resolved in our simulations, are supporting the robustness of our simulations despite the large values of $L_{x}$ used. Regarding the validity of the local approximation, global linear simulations have been carried out for few cases in order to test the effect of the variation of the gradients along $x$ on the linear growth rate, frequencies and $\phi-T$ cross phases. They have been found in good agreement with the ones from local linear and nonlinear simulations. Furthermore, considering that in our case $L_{\perp}=L_{T e} \sim 1 / 120, L_{T e}$ being the scale length of the parameter with the steepest gradient, and considering the results obtained in Ref.[37, 38, 39] (based on the Cyclone Base case, adiabatic electrons and in the electrostatic approximation), for our cases, an overestimation of the fluxes from local simulations of only $\sim 15 \%$ can be expected with respect to global simulations. A new release of the global electromagnetic version of the code, stable even at high $\beta$ values, is in preparation and should be available soon. It will then be possible to compare the results of this work with global simulations. Another aspect to take into account in simulations of the edge region is the high collision frequency. In order to have stable results even at very high level of collisionality, the 'Landau-Boltzmann' operator in GENE has been used. Some of the linear results have been tested against the new 'Sugama' collision operator [40], recently implemented in GENE [41], founding an almost perfect agreement with the Landau-Boltzmann operator in our range of parameters.

The gyrokinetic analysis has been carried out at $\rho_{\text {tor }}=0.925$ for the ASDEX Upgrade discharges and at $\rho_{\text {tor }}=0.95$ for the JET discharges, where $\rho_{\text {tor }}=\sqrt{\Phi / \Phi_{\max }}$, $\Phi$ being the toroidal magnetic flux. The main experimental plasma parameters used as input in the simulations are listed in Table 2. The pressure, temperature and density normalized gradient lengths reported in this paper are calculated using the plasma major radius $\left(R_{0}\right)$ as normalization length and the gradients computed using $r=\left(R_{\text {out }}-R_{\text {in }}\right) / 2\left(R_{\text {out }}\right.$ and $R_{\text {in }}$ being the outer and the inner mid-plane major radius) as radial coordinate. The length used to normalize all the inputs (such as $\nu_{e i}, \gamma_{E x B}, \ldots$ ) and the outputs (such as the $\left.\gamma, \omega, \ldots\right)$ of the simulations is $\rho=\sqrt{\left(\Phi / \pi B_{\text {ref }}\right)_{\text {max }}}$, where $B_{\text {ref }}$ is the magnetic field at the center of the plasma. $\rho \approx 0.65 \mathrm{~m}$ for ASDEX Upgrade and $\rho \approx 1.15 \mathrm{~m}$ for JET $\left(R_{0} / \rho \approx 2.5\right.$ for both ASDEX Upgrade and JET). Other normalization quantities are the sound velocity $c_{s}=\sqrt{T_{e} / m_{i}}$ and the sound Larmor radius $\rho_{s}=\sqrt{T_{e} m_{i}} / e B_{r e f}$, where $m_{i}$ is the mass of the ions in the simulation $(H, \mathrm{D}, \ldots$ mass in $\mathrm{H}, \mathrm{D}, \ldots$ simulations). The gyro-Bohm normalization, $q_{e / i, g B}=q_{e / i} \cdot e^{2} B^{2} \rho^{2} /\left(n_{e} T_{e}^{1.5} \sqrt{m_{i}}\right)$, $q_{e / i}$ being the electron/ion heat flux in $W / m^{2}$, is used for the heat fluxes. These normalization quantities have been chosen in order to easily recognize deviations from the gyro-Bohm scaling. A pure gyro-Bohm scaling would predict the same normalized fluxes and normalized growth rates for every hydrogen isotope. In this way different normalized fluxes or growth rates between $H$ and $D$ in the plots are easily identified as deviation form the gyro-Bohm scaling. The time-traces of $q_{i, g B}$ of some of the simulations for the ASDEX Upgrade case are shown in Fig.3.

\section{Gyro-kinetic analysis}

In this section the results from the gyrokinetic simulations are discussed. In order to avoid repetitive plots in the figures, when similar results are obtained between JET-ILW and ASDEX Upgrade, only the results for ASDEX Upgrade are shown. 


\subsection{Characterization of the edge instability}

The normalized linear growth rate $\left(\gamma\left(\rho / c_{s}\right)\right)$ and the normalized frequency $\left(\omega\left(\rho / c_{s}\right)\right)$ of the most unstable modes in the linear simulations are shown in Fig.4a and in Fig.4b as a function of $k_{y} \rho_{s}$. For the same plasma parameters, in the spectral range that determines the non-linear fluxes, i.e. $k_{y} \rho_{s} \lesssim 0.3, H$ has a higher growth rate with respect to $D$ (up to $+80 \%$ at $\left.k_{y} \rho_{s}=0.15\right)$. This result, valid in both electrostatic and electromagnetic linear simulations, indicates a deviation from the gyro-Bohm expectations. When the different value of $R / L_{n}$ between $H$ and $D$ plasmas is considered, the growth rate in the $\mathrm{D}$ simulation increases in the low $k_{y}$ region and decreases for $k_{y} \rho_{s}>0.3$ for ASDEX Upgrade. The effect of $R / L_{n}$ is less important for JET, where it does not change, between $\mathrm{H}$ and $\mathrm{D}$ plasmas, by the same amount as in the ASDEX Upgrade cases. As it will be shown in section 4.4, in our range of parameters, the destabilizing effect of $R / L_{n}$ at low $k_{y}$ is the dominant one in the nonlinear simulations. In both devices, the dominant linear mode in the range $0.1 \lesssim k_{y} \rho_{s} \lesssim 0.3$ is a drift-wave-like mode with negative real part of the frequency $\omega$ (Fig.4b), indicating that it rotates in the electron diamagnetic direction. The drift-wave nature of the instability appears from the phase-shift angle $\alpha$ between the electrostatic potential $(\phi)$ fluctuations and the electron temperature fluctuations $\left(\tilde{T}_{e}\right)$, shown in Fig.5. $\alpha<\pi / 2$, as typical of drift waves, while, for interchange instabilities like the Resistive Ballooning Modes, $\alpha=\pi / 2$. The ballooning structures of $\phi$ and $A_{\|}$are shown in Fig.6. $\phi$ has a ballooned structure with tails and $A_{\|}$is asymmetric with a minimum at the ballooning angle $\theta=0$. These observations, in addition to the fact that electrostatic simulations give similar results with respect to the electromagnetic simulations, exclude micro tearing modes (MTM) and other electromagnetic instabilities such as kinetic ballooning modes (KBM). These properties of the instability are confirmed by the global linear simulations and by the non-linear local simulations, as shown in Fig.4b and in Fig.5. A difference between the linear and the non-linear results is that, while in the linear simulations the drift-wave instability with negative $\omega$ is dominant for $k_{y} \rho_{s} \gtrsim 0.1$, in the non-linear simulations the turbulence is dominated by an electron drift-wave instability also at lower $k_{y}$. The fact that these fundamental properties of the linear instability are valid also in the non-linear simulations (at least in the range of $k_{y}$ where the flux spectra peaks) is a promising sign for possible future quasi-linear approximations for the turbulent flux in the edge. However, as will be shown in the next sections, a non-linear electromagnetic effect can play a key role for the fluxes and must be somehow taken into account in future quasi-linear models.

Another important property of the linear instability is that it is characterized by a mass dependent threshold in $R / L_{T e}$, the threshold being lower with lower isotope mass (left plot of Fig.7a). This is valid for both JET-ILW and ASDEX Upgrade. The linear threshold in $R / L_{T e}$ has been studied for ASDEX Upgrade at different $k_{y} \rho_{s}$ $(=0.12,0.16,0.2)$ and at different $\beta_{e}\left(=10^{-6}, 2.2 \cdot 10^{-4}, 4.4 \cdot 10^{-4}\right)$ using $R / L_{T i}=R / L_{n}=0$ and the results are shown in Fig.7a (right plot) and in Fig.7b. Two aspects of the instability emerge. For the considered experimental parameters, the electron drift-wave survives linearly at lower values of $k_{y}$ in $H$ than in D. This already emerged from the frequencies and the phase angles (Fig.4b and Fig.5). When looking at the effect of $\beta_{e}$, it appears that $\beta_{e}$ affects the linear threshold of the electron drift-waves when finite values of $R / L_{T i}$ are considered while, when $R / L_{T i}=0$, its effect on the threshold is negligible. This is visible comparing the right and the left plots of Fig.7a. At $k_{y}=0.12,0.16$ (Fig.7b), a competition between the drift-wave-like modes and MTM appears when increasing $\beta_{e}$, this effect being stronger going at lower $k_{y}$. In H, MTM are stable at the experimental values of $\beta_{e}$ and appears only when increasing its value. In D, the MTM linear threshold is lower and these modes appear also at values of $\beta_{e}$ close to the experimental ones. In agreement with these linear results, MTM are found in D non-linear simulations at the nominal values of $\beta_{e}$ while they are not found in $\mathrm{H}$ simulations for both JET-ILW and ASDEX Upgrade. The linear effects of $T_{i} / T_{e}$ and $R / L_{n}$ on the instability have also been studied and scans in these parameters are shown in Fig.8. It appears that the effects of both $R / L_{n}$ and $T_{e} / T_{i}$ on these modes have a non-monotonic behavior but are similar with different isotope mass. 
No strong dependence on $R / L_{T i}$ has been observed in the linear simulations.

\subsection{Effects of collisionality}

A key aspect of the edge region of L-mode plasmas is collisionality. The collision frequency is very high in the edge region of the studied discharges and has a strong impact on the nature of the turbulence. A linear scan in the collisionality $\nu_{e}^{*}=\nu_{e i} \cdot 4 / 3 \sqrt{\pi} \cdot q R^{2} /\left(\epsilon^{1.5} \cdot v_{t h, e}\right)$, where $v_{t h, e}=\sqrt{T_{e} / m_{e}}, \epsilon=r / R$ and $q$ is the safety factor, using $k_{y} \rho_{s}=0.2$, is shown in Fig.9a. Collisions stabilize at first but, beyond a certain value of $\nu_{e}^{*}$ corresponding to a minimum in the linear growth rate $\gamma\left(\rho / c_{s}\right)$, they have a destabilizing effect. The experimental $\nu_{e}^{*}$ of the studied discharges lies in the region close to the minimum in the growth rate where collisions are destabilizing. If the same scan in $\nu_{e}^{*}$ is done using adiabatic passing electrons (using the method described in Ref.[31]), collisions have always a stabilizing effect, as shown by the black stars in Fig.9a. This result indicates that the dynamics of passing kinetic electrons is fundamental for the instability at high collisionality. The importance of the passing particles and the less important role of trapped particles can also be deduced by the plots of Fig.10. In Fig.10a the behavior of the linear growth rate with the fraction of the trapped particle is studied using an $s-\alpha$ geometry and varying the parameter $\epsilon=r / R$. This corresponds to a scan in the trapped particle fraction $f_{t}[42,43]$. Within an approximation of adiabatic passing electrons, the linear growth rate increases beyond a certain threshold of the trapped particle fraction, as expected for TEM or for dissipative trapped electron modes (DTEM). Instead, when considering kinetic passing electrons, the instability is present also at $\epsilon=0$ and the growth rate does not strongly depend on the trapped particle fraction. In Fig.10b, following the methods used in Ref. [44, 45, 46, 47], the contribution to the growth rate of the electron curvature term $\gamma_{c u r v}^{e l}$, that is the main linear drive of the instability, is shown in the velocity-space $\left(v_{\|}, \mu\right)$. At low collisionality (left plot, $\left.\nu_{e}^{*}=0.0368\right)$, the trapped particle region is fundamental for the instability and $\gamma_{c u r v}^{e l}$ is determined by the contribution from this region. At the experimental level of collisionality (right plot, $\nu_{e}^{*}=3.68$ ), the dominant region that contributes to $\gamma_{c u r v}^{e l}\left(v_{\|}, \mu\right)$ is the passing particle region. Within our range of parameters, collisions cause the change from a trapped-particle instability (such as TEM or DTEM) to an instability dominated by the passing electron dynamics.

Collisions also influence the effect of the isotope mass on the instability. From Fig.9a it appears that the difference in growth rate between $\mathrm{H}$ and $\mathrm{D}$ increases with collisionality, reaching a maximum around the location of the minimum in growth rate. Collisionality is also determining the isotope mass effect on the eigenfunctions of the electrostatic potential $\phi(z)$, shown in Fig.11a for $\nu_{e}^{*}=0.0368$ and in Fig.11b for $\nu_{e}^{*}=3.68$. While at low collisionality $\phi(z)$ does not change substantially with the isotope mass, at high collisionality it has broader structures with lower isotope mass. It is important to notice that this effect is not related to a change of the effective collisionality $\left(\nu_{e i} / \omega_{t, i} \propto \sqrt{m_{i}}\right.$, where $\omega_{t, i}$ is the ion transit frequency [9]), as appeared from dedicated simulations, but is related to the change of the isotope mass itself, pointing to a role of $m_{e} / m_{i}$ when the electron parallel dynamics is strongly damped by collisions. The contributions from the different terms in the linear gyrokinetic equations, i.e. the term related to the parallel motion $\left(\gamma_{\|}\right)$and the term related to the $\nabla \mathbf{B}$ and to the B-curvature drifts $\left(\gamma_{\text {curv }}\right)$ (the term related to collisions $\left(\gamma_{\text {coll }}\right)$ is negligible and is not shown), of the different species to the overall micro-instability growth rate $\left(\gamma_{t o t}\right)$ have been investigated in the velocity space $\left(v_{\|}, \mu\right)$ and in the $\left(z, v_{\|}-\mu\right)$ space and are shown in Fig.12a at different collisionalities. The stabilizing effect of the electron parallel dynamics (negative contribution) is strongly reduced at high collisionality, at a higher rate than the reduction of the curvature terms. When the collisionality is increased, the various terms start to show an increasing difference between $\mathrm{H}$ and $\mathrm{D}$, the contribution to the growth rate from the ion curvature term exhibiting the largest difference. This because the larger structures of $\phi(z)$ with lower isotope mass influence the contribution of the curvature term of both species but in particular that of the ions (Fig.12b). The effect of the isotope mass at high collisionality translates in the higher $R / L_{T e}$ threshold and in the lower growth rate 
with higher isotope mass. We remark that, at low collisionality, no substantial differences in $\phi(z)$ and in the linear threshold have been observed.

A two point scan in collisionality has been performed also with non-linear simulations for the ASDEX Upgrade case and is shown in Fig.9b. At low collisionality the ion heat flux follows the gyro-Bohm scaling within error bars in both the electrostatic and in the electromagnetic simulations. At the experimental value of $\nu_{e}^{*}$, the fluxes exhibit a strong deviation from the gyro-Bohm scaling, with the normalized $H$ fluxes higher than the $D$ fluxes both in the electrostatic and in the electromagnetic simulations, with the electromagnetic effects enhancing the deviation from the gyro-Bohm scaling at high collisionality. The non-linear simulations confirm also what found linearly for $\phi(z)$. In Fig.13 the structures of $\phi(z)$ for $k_{y} \rho_{s}=0.2, k_{x}=0$ and averaged in time from the nonlinear electrostatic simulations are shown. As in linear simulations, at low collisionality no strong deviations between $H$ and $D$ is visible while at high collisionality the structure of $H$ are broader than the ones of $D$.

All the observations reported above in this sub-section could be summarized as this: the high collisionality in the plasma edge determines the strong non-adiabatic response of the electrons to the variations of the electrostatic potential $\phi$ fluctuations and so the destabilization of the electron drift-waves found to be dominant in our linear simulations. The passing electron dynamics, being strongly damped by collisions, play a key role for the instability and the fact that the passing electron dynamics is highly non-adiabatic strongly influence the role of the isotope mass in the destabilization of the drift-waves. However, a simple and complete analytical explanation of the role of the isotope mass is missing at the moment.

\subsection{Electromagnetic effects}

As anticipated in sections 4.1 and 4.2, electromagnetic effects are found to destabilize the turbulence in both linear and nonlinear simulations but with remarkably different degrees. This is visible in Fig. 9b and in Fig.14, where a scan of the linear growth rate in $\beta_{e}$ using $k_{y} \rho_{s}=0.15$ is shown for both ASDEX Upgrade and JET and a scan in $q_{i}\left(\mathrm{~kW} / \mathrm{m}^{2}\right)$ is shown for ASDEX Upgrade. In linear simulations electromagnetic effects are destabilizing but this effect is not very strong until the kinetic ballooning modes (KBM) threshold is reached. Instead, when electromagnetic effects are included in the non-linear simulations, a strong enhancement of the fluxes is observed at values of $\beta_{e}$ much lower than those predicted from linear simulations. Looking at the $k_{y}$ spectra of the electron heat flux, shown in Fig.15a for ASDEX Upgrade and in Fig.15b for JET-ILW, in the electromagnetic simulations there is a strong enhancement of the contribution to the turbulent flux from the low- $k_{y}$ wave-numbers and a shift of the peak of the flux spectra to lower $k_{y}$ with respect to electrostatic simulations. Accordingly, as shown in Fig.16, when increasing $\beta_{e}$, the peak of the cross-phase between the electrostatic potential and the electron temperature fluctuations moves to lower $k_{y}$ and to values of $\alpha\left(\phi-\tilde{T}_{e, \perp}\right)$ closer to $\pi / 2$. These observations, in agreement with the results of Ref. [29, 24], suggest that a transition to MHD-like turbulence occurs nonlinearly at values of $\beta_{e}$ much lower than those expected from the linear simulations. We observe that the parameter $\hat{\beta}$ could be used to parametrize such transition. In our cases, as already observed in past studies (see e. g. Ref.[29]), $\hat{\beta} \approx 1$ is close to the non-linear threshold in $\beta_{e}$ and could be use as an indicator for the electromagnetic non-linear effects to start to play an important role. Looking at Fig.9b, other interesting aspects of the electromagnetic effects emerge. First, the effect of $\beta_{e}$ is influenced by collisionality. In both linear and nonlinear simulations the increase in $\gamma$ and $q_{i, g B}$ is stronger at low collisionality (at $\nu_{e}^{*}=0.0368, q_{i, g B}^{e m} \sim 6.4 q_{i, g B}^{e s}$ while, at $\nu_{e}^{*}=3.68, q_{i, g B}^{e m} \sim 3.8 q_{i, g B}^{e s}$ for $\mathrm{H}$ and $q_{i, g B}^{e m} \sim 2.8 q_{i, g B}^{e s}$ for D). Second, for the same parameters, the enhancement of the fluxes at high collisionality is stronger in $H$ than in $\mathrm{D}$. This can be related to the fact that, for the same value $\beta_{e}$, the drift-wave instability has a lower linear threshold in $\mathrm{H}$ than in $\mathrm{D}$ at high collisionality while the linear threshold does not change substantially at low collisionality. Also the fact that the dynamics of passing kinetic electrons is fundamental at high collisionality and that it 
is influenced by $m_{e} / m_{i}$ could play a role in determining the difference of the strength of the electromagnetic effects between $\mathrm{H}$ and D in non-linear simulations. Despite the fact that electromagnetic effects can be dominant nonlinearly, it is important to notice that the fluxes are, in all cases, determined by the electrostatic potential fluctuations.

A final remark on the non-linear simulations is that, when using the nominal experimental parameters ( $\beta=2.4 \cdot 10^{-4}$ and $R / L_{T e}=47$ for ASDEX Upgrade and $\beta=2.2 \cdot 10^{-4}$ and $R / L_{T e}=80$ for JET) in D simulations, MTM modes appears for both devices. MTM were observed in the H simulations. This difference can be related to the higher effective collisionality in $\mathrm{D}$ with respect to $\mathrm{H}$. This would be consistent with the fact that, for the JET case, MTM disappear when the collision frequency is slightly decreased. With unstable MTM, the electromagnetic electron heat flux reached values one order of magnitude higher that the experimental values and so the parameters were adjusted within error bars to avoid them and reduce the fluxes. Nevertheless, the fact that they are predicted for parameters within the experimental range indicates that they could also play an important role in limiting the electron temperature of L-mode plasmas.

\subsection{Zonal-flow activity}

In order to investigate a possible role of zonal-flows in the isotope effect and/or in the electromagnetic effect observed in the simulations, the ratio between the instability linear growth rate and the zonal flow shearing rate as well as the ratio between the zonal and the non-zonal components of the electrostatic potential have been analyzed. The first quantity is $\gamma / \omega_{E}$, where $\omega_{E}=\frac{d}{d x} V_{E, y}\left(k_{y}, k_{\|}=0\right)\left(V_{E, y}\right.$ being the $E \times B$ velocity related to the zonal flows), and gives an estimate on the effectiveness of zonal flow in saturating the linear instability. The second quantity is $\phi^{z f} / \phi^{\text {turb }}=\phi\left(k_{y}=0\right) / \sum_{k_{y} \neq 0} \phi\left(k_{y}\right)$, where $\phi\left(k_{y}\right)$ indicates the potential at a certain value of $k_{y}$ averaged over $z$ and $k_{x}$, and is an indicator of the strength of the zonal flow compared to the strength of the turbulence. The values of these quantities in the simulations shown in Fig.9b and in the $H$ simulations with doubled $\beta$, i.e. with $\beta_{e}=3.6 \cdot 10^{-4}$, are reported in Table 3. In the low collisionality case no relation between the zonal flow activity and the trend of the fluxes with the electromagnetic effect has been found. In the high collisionality case, slightly lower values of $\phi^{z f} / \phi^{\text {turb }}$ for $\mathrm{H}$ with respect to $\mathrm{D}$ are found in the electromagnetic case while a much lower value is found in the electrostatic case. For H also a slight increase of $\gamma / \omega_{E}$ is observed when increasing $\beta_{e}$, but this is not observed for D. Overall, despite these trends, no clear relation between the zonal-flow activity and the electromagnetic effects and between the zonal-flow activity and the fluxes behavior with the isotope mass has been identified in the simulations.

\subsection{Comparison with the experiment}

The comparisons between the experimental fluxes in $W / \mathrm{m}^{2}$ and the gyrokinetic predictions for ASDEX Upgrade and JET-ILW are shown in Fig.17. For ASDEX Upgrade a separation of the electron and the ion heat fluxes was possible and the results are shown in Fig.17a for $q_{i}$ and in Fig.17b for $q_{e}$, while, for JET, only the total heat flux could be determined experimentally and the comparison is shown in Fig.17c. For both devices the heat fluxes from the electrostatic simulations are in good agreement with the experiment. When electromagnetic effects are included in the simulations, due to the strong enhancement of the fluxes related to the electromagnetic effects, $\beta_{e}$ and $R / L_{T e}$ had to be lowered within error-bars in order to recover the experimental fluxes. This is especially true for ASDEX Upgrade, where a reduction of $\beta_{e}$ from $2 \cdot 2 \cdot 10^{-4}$ to $1.8 \cdot 10^{-4}$ and a reduction of $R / L_{T e}$ from 47 to 39 was necessary. For JET-ILW the main change in the parameters regards a reduction of the collisions frequency (of $\sim 5 \%$ ) in order to avoid MTM in the $D$ simulations that would give extremely high electron heat fluxes. Regarding the effect of the different values of $R / L_{n}$ between $\mathrm{D}$ and $\mathrm{H}$ plasmas, when the higher $R / L_{n}$ is used in D simulations, the fluxes increase for ASDEX Upgrade (magenta stars on the left plots of Fig.17a and 17b), following the destabilization at $k_{y} \rho_{s} \lesssim 0.25$ found linearly (Fig.4a). When considering the external flow 
shear in the $H$ simulation of ASDEX Upgrade, the heat flux is reduced even at the low experimental level of $\gamma_{E x B}$ (green stars in the left plots of Fig.17a and 17b). This result indicates that the edge turbulence is affected by an imposed $E \times B$ shear, supporting the hypothesis of a role of $\gamma_{E x B}$ in suppressing the edge turbulence and in helping the formation of the edge transport barrier that leads to the L-H transition. A deeper study on the competition between the equilibrium $E \times B$ shear rise (mainly due to $R / L_{T i}$ and $R / L_{n}$ in the diamagnetic term of the equilibrium radial electric field) and the linear drive of the turbulence $\left(R / L_{T e}\right.$ and, depending on its values, $R / L_{n}$ ) must be carried out in order to draw more solid results on this aspect. Finally, as for the heat fluxes, the particle fluxes $(\Gamma)$ are higher in $\mathrm{H}$ simulation with respect to D simulations in both the electrostatic and in the electromagnetic cases and for both devices (in Fig.18 the fluxes are shown for ASDEX Upgrade). The plot of Fig.18 also shows the strong deviation from the gyro-Bohm mass scaling found in the edge. This strong deviation, with the normalized fluxes of $\mathrm{H}$ much higher than the ones in $\mathrm{D}$ for the same set of parameters, is present already in the electrostatic simulations and is enhanced in the electromagnetic simulations. As for the heat flux in $\mathrm{H}$, the particle flux is influenced by the imposed $E \times B$ (green diamond in the plot) and, when considering the higher density gradient in D, it strongly increases (magenta star in the plot).

\section{Discussion and conclusions}

The nature of the turbulent transport in the edge of ASDEX Upgrade and JET-ILW L-modes has been investigated through linear and local non-linear gyrokinetic simulations. For both devices the turbulence has been found to be dominated by an electron drift-wave destabilized by the high collisionality in the plasma edge and strongly related to the dynamics of the kinetic passing electrons. This result, which is obtained here with specific parameters of ASDEX Upgrade and JET-ILW plasmas, confirms past general edge turbulence studies and simulations $[29,24,30]$. The related linear instability features an isotope mass dependent threshold in $R / L_{T e}$, the threshold being lower with lower isotope mass, and a non-monotonic dependence on $R / L_{n}$ and on $T_{e} / T_{i}$. No strong effect of $R / L_{T i}$ has been found. The effect of the isotope mass on the threshold is related to broader eigenfunctions of $\phi(z)$ that induce a larger contribution of the curvature terms, and especially of the ion curvature term, to the linear growth rate with lower isotope mass. This effect takes place only at high value of collisionality, while, at levels typical of the plasma core, this effect is negligible. This result supports the role of the collisionality in damping the parallel electron dynamics allowing the ion mass to have a stronger effect on the instability. These results are confirmed by the non-linear simulations where higher gyro-Bohm normalized fluxes are found in $\mathrm{H}$ with respect to D simulations at high collisionality while, at low collisionality, consistency with the gyro-Bohm expectations is found for the fluxes. Linear and non-linear simulations have been also found to agree with each other regarding some of the characteristics of the turbulence, such as the real part of the frequency $\omega\left(\rho / c_{s}\right)$ and cross-phase angle between the electrostatic potential and the electron pressure fluctuations. This is encouraging for next development of quasi-linear models for the edge turbulence. The turbulence in the edge is found to be strongly influenced by electromagnetic effects. Consistently with the results presented in Ref. $[24,30]$, this clearly appears in the non-linear simulations, where a strong enhancement of the contribution to the turbulent fluxes from low $k_{y}$ wave-numbers is seen when electromagnetic effects are included, while it is not observed in linear simulations. As identified in Ref.[24, 30], and as also demonstrated in this work by the effect of increasing $\beta_{e}$ on the cross-phases angle and on the $k_{y}$ flux spectra, these strong electromagnetic effects in non-linear simulations can be related to the fact that larger structures in $\phi$, with more MHD-like properties, become dominant when increasing $\beta_{e}$ at values well below the linear MHD limit. This aspect can be relevant when trying to compute the turbulent fluxes by means of quasi-linear models. Looking at the results in Ref. [24, 30], a threshold in the normalized parameter $\hat{\beta}=\beta_{e}\left(\hat{q} R / L_{\perp}\right)^{2}$ can be identified for the importance of electromagnetic effects in nonlinear simulations, i.e. $\hat{\beta} \gtrsim 1$. In the present ASDEX Upgrade and JET-ILW cases $\hat{\beta}>1$, and also in our cases $\hat{\beta} \approx 1$ is close to the non-linear threshold in $\beta_{e}$ for the electromagnetic effects 
to be important. This observation support what already pointed out in Ref.[29]), i.e. that $\hat{\beta} \approx 1$ could be use as an indicator for the electromagnetic non-linear effects to start to play an important role in the edge. Even if the saturated turbulent fluxes peak in the region $0.1 \lesssim k_{y} \rho_{s} \lesssim 0.2$, where the linear characteristics of the turbulence survive in the nonlinear simulations, a discrepancy between linear and nonlinear results is already visible (Fig.14), due to the role of structures at $k_{y} \rho_{s} \lesssim 0.1$ in the nonlinear flux spectra (Fig.15). For higher values of $\hat{\beta}$, the role of the structures at $k_{y} \rho_{s} \lesssim 0.1$ becomes more and more dominant and the discrepancy between linear and nonlinear results increases. All these elements are in line with the conclusions of Ref. [24, 30], i.e. when electromagnetic effects become dominant, the saturated turbulence in the plasma edge region cannot be directly determined using a linear approximation as, in contrast, is possible for a wide range of parameters in core plasma conditions (see e.g. $[48,49,50,51]$ ). The importance of the large structures at low $k_{y}$ in nonlinear simulations starts at $\beta$ levels well below the ones predicted by linear simulations and the electromagnetic effects are found to be stronger in $\mathrm{H}$ than in $\mathrm{D}$ at the same level of $\beta_{e}$. The combination of higher heat and particle transport in $\mathrm{H}$ plasmas, limiting temperature and density gradients, also leads to the requirement of a higher separatrix density to reach the same line averaged density, in agreement with the experimental observations. This can indeed play an important role in determining the higher L-H power threshold in $\mathrm{H}$ plasmas with respect to D plasmas. Also, the turbulent fluxes have been found to be sensitive to the imposed $E \times B$ flow shear, even for the low experimental level of $\gamma_{E x B}$, supporting the hypothesis of a role of the $\gamma_{E x B}$ driven by the radial electric field in suppressing the edge turbulence helping the formation of the external transport barrier and the L-H transition. These results support the hypothesis, already expressed for the JET-ILW cases in Ref. [3], that the turbulent transport plays a crucial role in explaining the different plasma edge behavior and the higher L-H power thresholds with different isotope masses. Remarkably, when considering all the effects in the non-linear simulations and the experimental uncertainties, the predicted fluxes can reproduce the experimental fluxes and the experimental behavior with the isotope mass.

Finally, we recall some remarks on the gyro-kinetic simulations in these plasma conditions. In our case $\rho^{*}=\rho_{s} / L_{\perp} \approx 1 / 120$, where we used $L_{\perp}=L_{T e}$, i.e. the scale length of the parameter with the steepest gradient. Considering the results obtained in Ref.[38, 39], based on the Cyclone Base case with $a / L_{T e}=2.3$, adiabatic electrons and in the electrostatic approximation, an overestimations of the fluxes from local simulations $\lesssim 15 \%$ can be expected with respect global simulations for our cases. Electromagnetic effects, plasma shape and kinetic electrons, all effects considered in our simulations, can also play a role in the deviation from global simulations [37]. The present global version of GENE is unstable when the magnetic fluctuations play an important role. However, a new global version of GENE, stable even at high $\beta$, is in the testing phase and it will eventually be possible to confirm the results of this work with the new version of the code. The appearance of radially large structures in $A_{\|}$can also create some problem in the interpretation of the non-linear results. In particular, non-physical fluctuations at the lowest toroidal mode numbers and with high frequencies $\left(\omega\left(\rho / c_{s}\right)>10\right)$ can appear in the simulation. We handled them with a large radial box size, but it is important to note that the results obtained with this method do not depend on the box size itself (the same fluxes were obtained using $L_{x}=120 \rho_{s}$ and $L_{x}=450 \rho_{s}$ ) and are in good agreement with past results obtained using smaller radial box sizes and Dirichlet boundary conditions in the radial direction [24,30]. This because, even if electromagnetic effects are important in the non-linear simulations, the fluxes and the main characteristics of the turbulence are found to be determined by the electrostatic potential structures and are not affected by the $A_{\|}$structures.

\section{Acknowledgement}

The authors are grateful to Alejandro Banon-Navarro and Tobias Goerler for their assistance with the GENE code and to Gabor Szepesi for his help with the JET equilibrium data. We would like to thank Clarisse Bourdelle and Gregory De Dominici for very useful discussion and suggestions. We acknowledge the CINECA 
award under the ISCRA initiative, for the availability of high performance computing resources and support. Part of the simulations presented in this work were performed at the COBRA HPC system at the Max Planck Computing and Data Facility (MPCDF), Germany. This work has been carried out within the framework of the EUROfusion Consortium and has received funding from the Euratom research and training programme 2014-2018 and 2019-2020 under grant agreement No 633053. The views and opinions expressed herein do not necessarily reflect those of the European Commission.

\section{References}

[1] M. Bessenrodt-Weberpals et al., Nucl. Fusion 33, 1205 (1993)

[2] C. W. Barnes et al., Phys. Plasmas 3, 4521 (1996)

[3] C. F. Maggi et al., Plasma Phys. Control. Fusion 60, 014045 (2018)

[4] S. D. Scott et al., Phys. Plasmas 2, 2299 (1995)

[5] G. Manfredi, M. Ottaviani, Phys. Rev. Lett. 79, 4190 (1997)

[6] B. D. Scott, Phys. Fluids B 4, 2468 (1992)

[7] J. Garcia et al., Nucl. Fusion 57 (2017) 014007

[8] P. Manas et al., accepted by Nucl. Fusion, 2018

[9] M. Nakata et al., Phys. Rev. Lett. 118, 165002 (2017)

[10] C. Angioni et al., Physics of Plasmas 25, 082517 (2018)

[11] Xu Y. et al., Phys. Rev. Lett. 110, 265005 (2013).

[12] Hahm T. S. et al., 2013 Nucl. Fusion 53, 072002

[13] Bustos A. et al., 2015 Phys. Plasmas 22, 012305

[14] Garbet X. and Waltz R. E., Phys. Plasmas 3, 1898 (1996).

[15] T. S. Hahm and P. H. Diamond, J. Korean Phys. Soc. 73, 747 (2018).

[16] F. Ryter et al., Nucl. Fusion 49, 062003 (2009)

[17] E. Righi et al., Nucl. Fusion 39, 309 (1999)

[18] Saibene G. et al., Nucl. Fusion 39, 1133 (1999).

[19] J.G. Cordey et al., Nucl. Fusion 39, 301 (1999)

[20] ITER report n. ITR-18-003, ITER Organization, 2018.

[21] D. R. Hatch et al., Nucl. Fusion 55, 063028 (2015)

[22] D. Told et al., Phys. Plasmas 15, 102306 (2008)

[23] D. R. Hatch et al., Nucl. Fusion 56, 104003 (2016)

[24] B. D. Scott, Phys. Plasmas 12, 062314 (2005) 
[25] A. Zeiler et al., Phys. Plasmas 3, 2951 (1996)

[26] C. Bourdelle et al., Plasma Phys. Control. Fusion 54, 115003 (2012)

[27] C. Bourdelle et al., Nucl. Fusion 54, 022001 (2014)

[28] B. Scott, Plasma Phys. Control. Fusion 34, 1977 (1992)

[29] B. D. Scott, Plasma Phys. Control. Fusion 49, S25 (2007)

[30] B. D. Scott, A. Kendl and T. Ribeiro, Contrib. Plasma Phys. 50, No. 3-5, 228 (2010)

[31] J. Dominski et al., Journal of Physics: Conference Series 401 (2012) 012006

[32] P. A. Schneider et al., Nucl. Fusion 57, 066003 (2017)

[33] Jenko F., et al., Phys. Plasmas 7, 1904 (2000).

[34] Görler T., et al., J. Comput. Phys. 230, 7053 (2011).

[35] E. A. Frieman and Liu Chen, Physics of Fluids 25, 502 (1982).

[36] Brizard A. J., Hahm T. S., Rev. Mod. Phys. 79, 421 (2007).

[37] J. Candy and R. E. Waltz, Phys. Rev Lett. 91, 045001 (2003)

[38] J. Candy, R. E. Waltz and W. Dorland, Phys. Plasmas 11, L25 (2004)

[39] B. F. McMillan et al., Phys. Rev. Letter 105, 155001 (2010)

[40] Sugama H., Watanabe T. H. and Nunami M., 2009 Phys. Plasmas 16112503.

[41] P. C. Crandall et al., "Multi-Species Collisions for Delta-F Gyrokinetic Simulations: Implementation and Validation with GENE", 2019, submitted to Computer Physics Communications.

[42] S. P. Hirshman, Physics of Fluids 31, 3150 (1988).

[43] Y. R. Lin-Liu ans R. L. Miller, Phys. Plasmas 2 (5), 1666 (1995).

[44] R. Hatzky et al., Phys. Plasmas 9, 898 (2002)

[45] P. Manas et al., Phys. Plasmas 22, 062302 (2015)

[46] A. Banon Navarro et al., Phys. Rev. Lett. 106, 055001 (2011)

[47] A. Di Siena et al 2018 Nucl. Fusion 58054002

[48] C. Bourdelle et al 2016 Plasma Phys. Control. Fusion 58014036.

[49] J. Citrin et al 2017 Plasma Phys. Control. Fusion 59124005

[50] P. Rodriguez-Fernandez et al 2018 Phys. Rev. Lett. 120075001

[51] Kinsey J. E. et al Phys. of Plasmas 15059082008 


\section{Tables}

\begin{tabular}{|c|c|c|c|c|c|}
\hline & $\rho_{\text {tor }}$ & $\hat{\beta}$ & $\hat{\mu}$ & C & $\nu_{B}$ \\
\hline AUG 30369 (D) & \multirow{2}{*}{0.925} & \multirow{2}{*}{2.6} & 3.4 & 1.1 & 0.07 \\
\hline AUG $31693(\mathrm{H})$ & & & 6.8 & 1.42 & 0.1 \\
\hline JET 89723 (D) & \multirow{2}{*}{0.95} & \multirow{2}{*}{3.2} & 7.5 & 0.82 & 0.038 \\
\hline JET $91450(\mathrm{H})$ & & & 15 & 1.14 & 0.052 \\
\hline
\end{tabular}

Table 1: Nominal experimental values of $\hat{\beta}, \hat{\mu}, C, \nu_{B}[28,29,24]$ (see also the introduction) for the studied JET-ILW and ASDEX Upgrade plasmas calculated using $L_{\perp}=L_{T e}$. These parameters are good indicators of the role of non-adiabatic passing electron response, electromagnetic effects and collisional effects in the plasma edge.

\begin{tabular}{|c|c|c|c|c|c|c|c|c|c|}
\hline & $\overline{T_{i} / T_{e}}$ & $\nu_{e}^{*}$ & $\hat{\nu}_{e i}$ & $\beta_{e}$ & $R / L_{T e}$ & $R / L_{T i}$ & $R / L_{n}$ & $s / \hat{q}$ & $\gamma_{E x B}$ \\
\hline AUG 30369 (D) & \multirow{2}{*}{1.3} & \multirow{2}{*}{3.68} & 6.35 & \multirow{2}{*}{$2.2 \cdot 10^{-4}$} & \multirow{2}{*}{47} & \multirow{2}{*}{33} & 31 & \multirow{2}{*}{$3.5 / 4.0$} & 0.055 \\
\hline AUG $31693(\mathrm{H})$ & & & 4.49 & & & & 20 & & 0.04 \\
\hline JET $89723(\mathrm{D})$ & \multirow{2}{*}{1.0} & \multirow{2}{*}{2} & 3.7 & \multirow{2}{*}{$2.1 \cdot 10^{-4}$} & \multirow{2}{*}{77} & \multirow{2}{*}{45} & 23 & \multirow{2}{*}{$3.8 / 3.6$} & \multirow{2}{*}{$\approx 0.1$} \\
\hline JET $91450(\mathrm{H})$ & & & 2.6 & & & & 17 & & \\
\hline
\end{tabular}

Table 2: Nominal experimental plasma parameters at $\rho_{\text {tor }}=0.925$ for ASDEX Upgrade and at $\rho_{\text {tor }}=0.95$ for JET-ILW. Here $\beta_{e}=\frac{8 \pi n_{e} T_{e}}{B_{r e f}^{2}}, \nu_{e}^{*}=\nu_{e i} \cdot \frac{4}{3 \sqrt{\pi}} \frac{q R^{2}}{\epsilon^{1.5}} \frac{1}{v_{t h, e}}, \hat{\nu}_{e i}=\nu_{e i} \cdot\left(\frac{\rho}{c_{s}}\right), \gamma_{E x B}=-\frac{\rho_{\text {tor }}}{q} \frac{\partial \Omega_{t}}{\partial \rho_{t o r}} \frac{\rho}{c_{s}}$, where $q$ is the safety factor, $\epsilon=\frac{r}{R}$, $\Omega_{t o r}$ is the angular toroidal velocity in $\frac{\mathrm{rad}}{\mathrm{s}}, v_{t h, e}=\sqrt{T_{e} / m_{e}}$ and $c_{s}=\sqrt{T_{e} / m_{i}}$.

\begin{tabular}{|c|c|c|c|}
\hline$\nu_{\mathbf{e}}^{*}=\mathbf{0 . 0 3 6 8}$ & $\beta_{e}$ & $\phi^{z f} / \phi^{\text {turb }}$ & $\gamma / \omega_{E}$ \\
\hline \hline $\mathrm{D}$ & $10^{-6}$ & 8.4 & 0.11 \\
\hline $\mathrm{D}$ & $1.8 \cdot 10^{-4}$ & 8.2 & 0.104 \\
\hline $\mathrm{H}$ & $10^{-6}$ & 8.3 & 0.123 \\
\hline $\mathrm{H}$ & $1.8 \cdot 10^{-4}$ & 7 & 0.117 \\
\hline$\nu_{\mathbf{e}}^{*}=\mathbf{3 . 6 8}$ & $\beta_{e}$ & $\phi^{z f} / \phi^{\text {turb }}$ & $\gamma / \omega_{E}$ \\
\hline \hline $\mathrm{D}$ & $10^{-6}$ & 9 & 0.056 \\
\hline $\mathrm{D}$ & $1.8 \cdot 10^{-4}$ & 4.8 & 0.057 \\
\hline $\mathrm{H}$ & $10^{-6}$ & 2.7 & 0.072 \\
\hline $\mathrm{H}$ & $1.8 \cdot 10^{-4}$ & 3.4 & 0.079 \\
\hline $\mathrm{H}$ & $3.6 \cdot 10^{-4}$ & 3.2 & 0.088 \\
\hline
\end{tabular}

Table 3: Values of the ratio between the instability linear growth rate and the zonal-flow shearing rate, $\gamma / \omega_{E}$, and of the ratio between the zonal and the non-zonal components of the electrostatic potential, $\phi^{z f} / \phi^{\text {turb }}$, in the ASDEX Upgrade simulations shown in Fig.9b and in the $H$ simulation with doubled $\beta_{e}$. Here $\phi^{z f} / \phi^{\text {turb }}=\phi\left(k_{y}=0\right) / \sum_{k_{y} \neq 0} \phi\left(k_{y}\right)$, where $\phi\left(k_{y}\right)$ indicates the potential at a certain value of $k_{y}$ averaged over $z$ and $k_{x}$, and $\omega_{E}=\frac{d}{d x} V_{E, y}\left(k_{y}, k_{\|}=0\right), V_{E, y}$ being the $E \times B$ velocity related to the zonal flows. 


\section{Figures}

a)
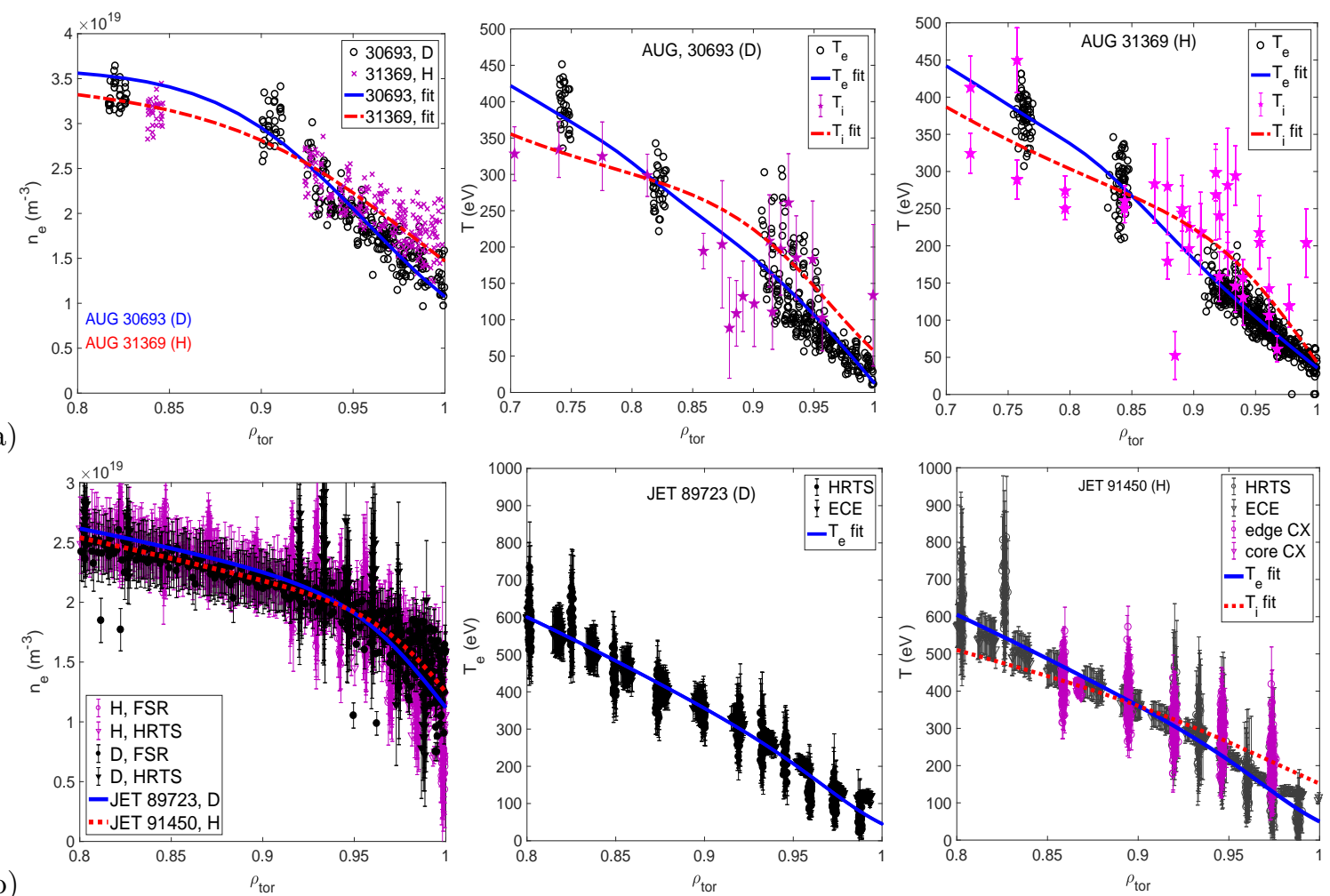

Figure 1: Fits of the experimental edge $T_{e}, T_{i}$ and $n_{e}$ profiles. (a) ASDEX Upgrade discharges \#30693 (D) and \#31369 (H); b) JET discharges \#89723 (D) and \#91450 (H). For ASDEX Upgrade, Thomson Scattering (TS) and Li-beam (LB) data were used for $n_{e}$, TS and Electron Cyclotron Emission (ECE) data for $T_{e}$ and Charge Exchange (CX) data for $T_{i}$. For JET, the High Resolution Thomson Scattering (HRTS) and the fast reflectometer (FSR) measurements were used for $n_{e}$, HRTS and ECE measurements where used for $T_{e}$ and the edge $C X$ and the core D $\alpha$ CX were used for $T_{i}$. As the experimental profile are very similar but the $C X$ data for the $D$ plasmas is poor, the same $T_{i}$ profile is used for the D and H JET plasmas. More details on these plasmas can be found in Ref.[32] for ASDEX Upgrade and in Ref.[3] for JET-ILW. 

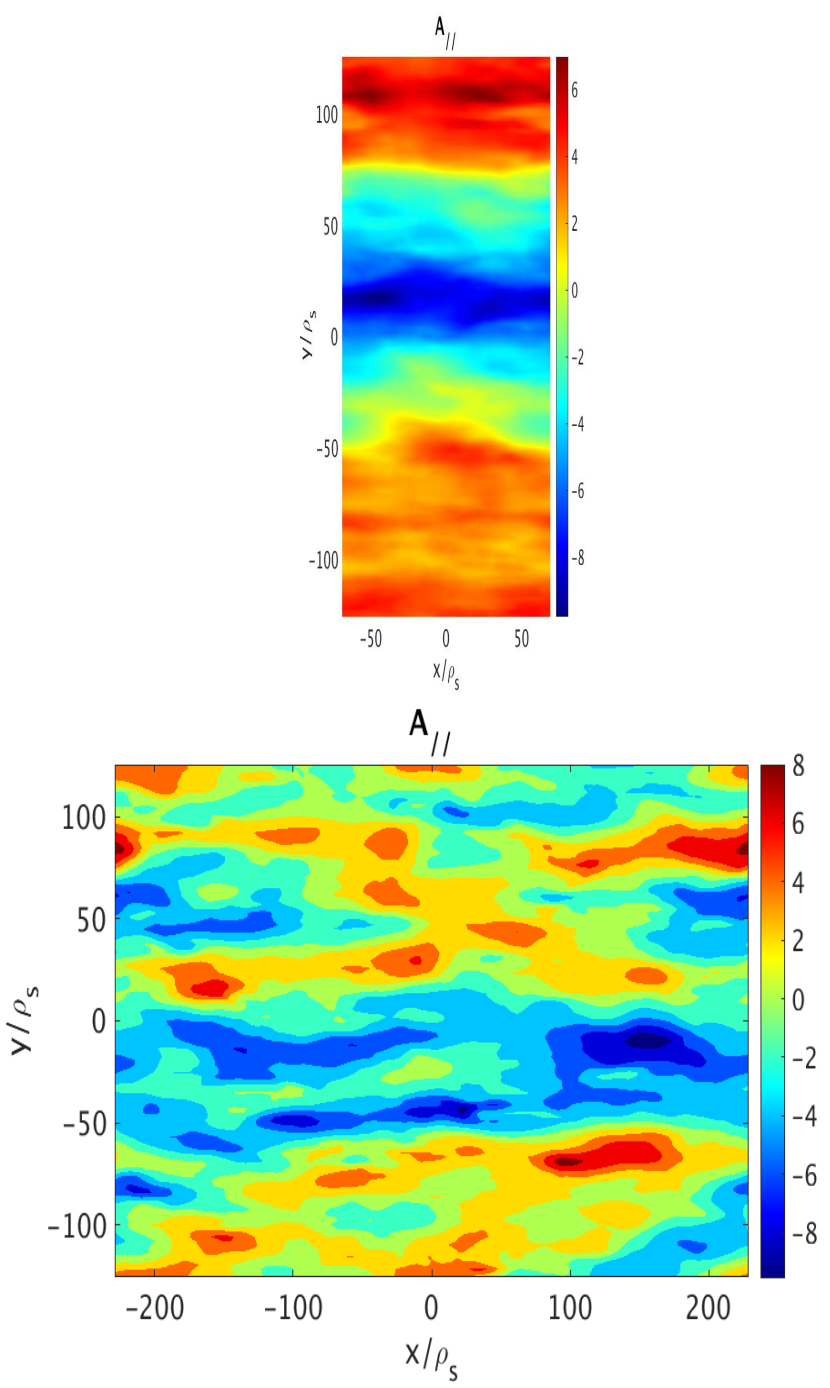
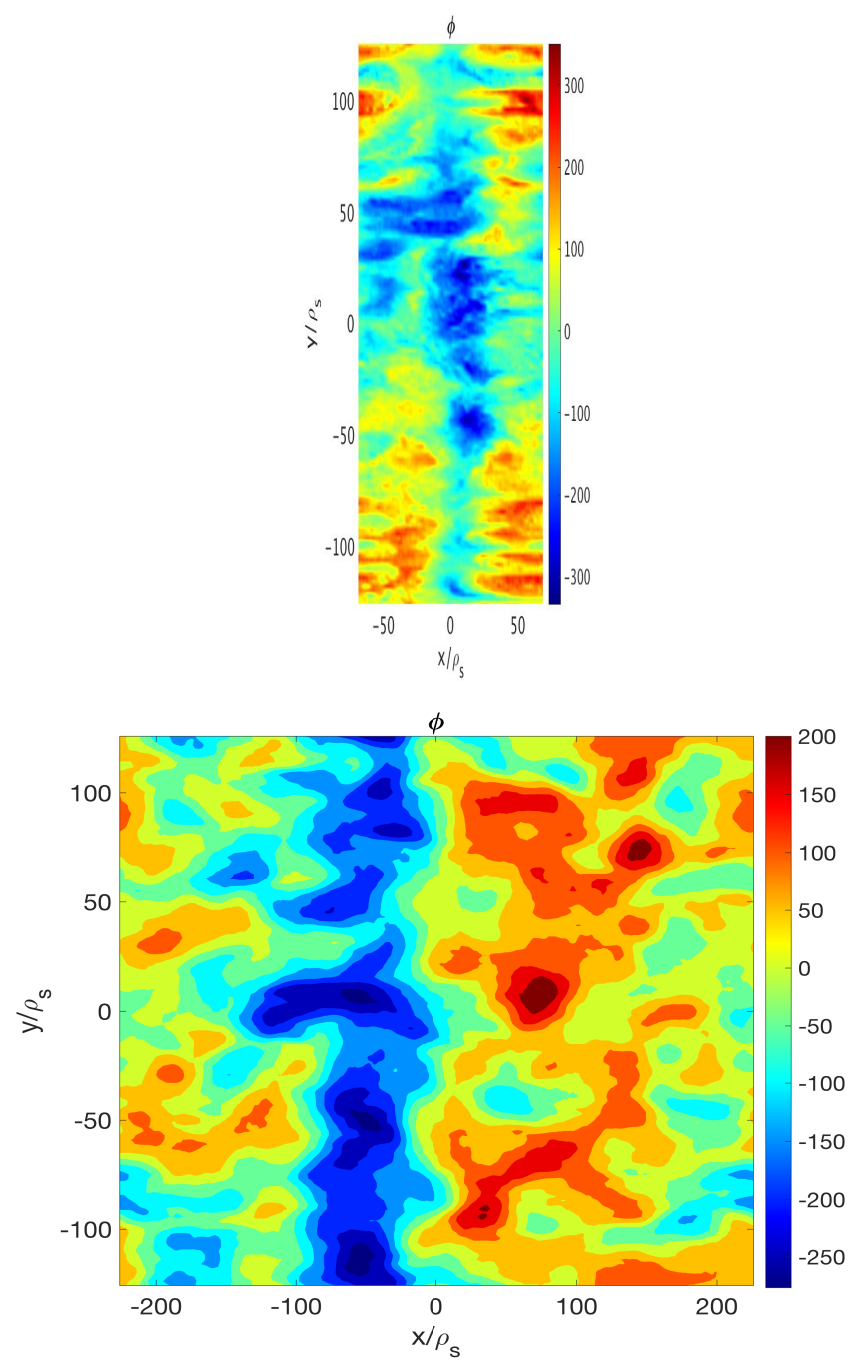

Figure 2: Contour plot of the electrostatic potential $\phi(x, y)$ and of the parallel magnetic potential $A_{\|}(x, y)$ from the non-linear gyrokinetic simulations with different radial box sizes. Above $L_{x} \approx 120 \rho_{s}$, below $L_{x} \approx 450 \rho_{s}$. While the electrostatic potential structures are resolved in both cases, when using a box resolution $L_{x} \lesssim 400 \rho_{s}$ the $A_{\|}$radially elongated structures cannot be resolved and unphysical frequencies appears at the lowest toroidal mode numbers in the simulation. 


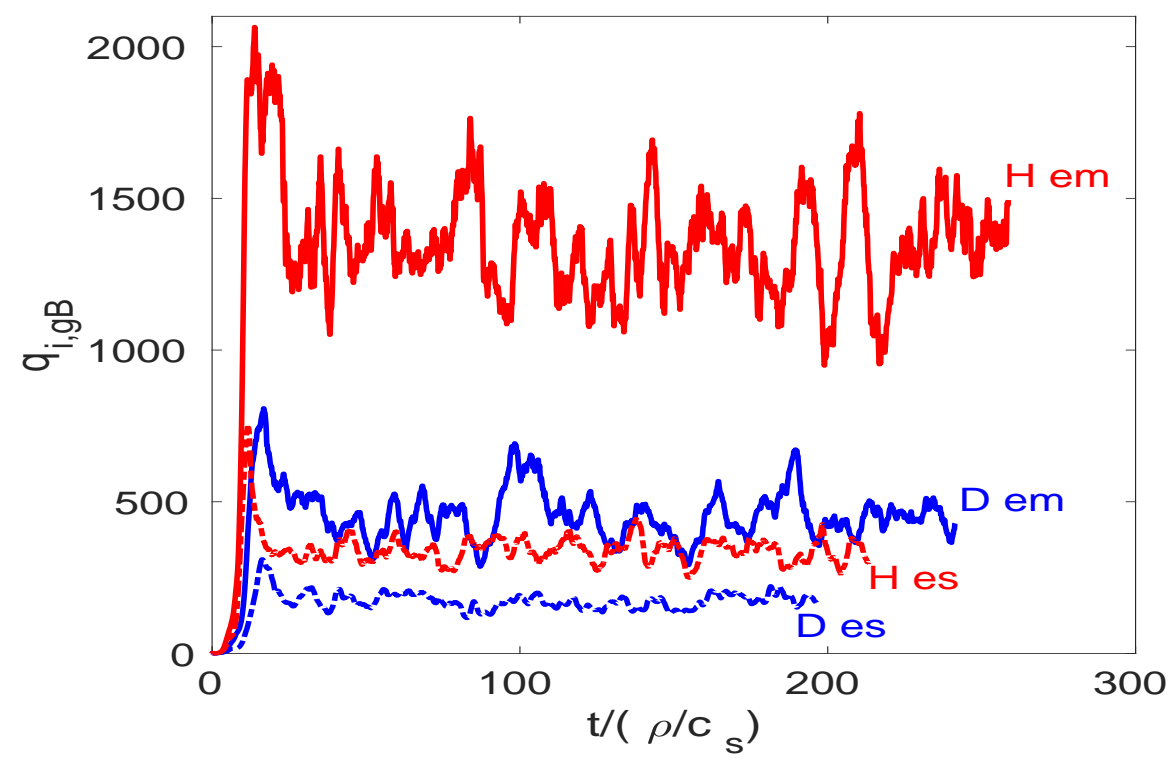

Figure 3: Time evolution of the gyro-Bohm normalized ion heat flux from some of the simulations of the ASDEX Upgrade discharge shown in Fig.17a. 


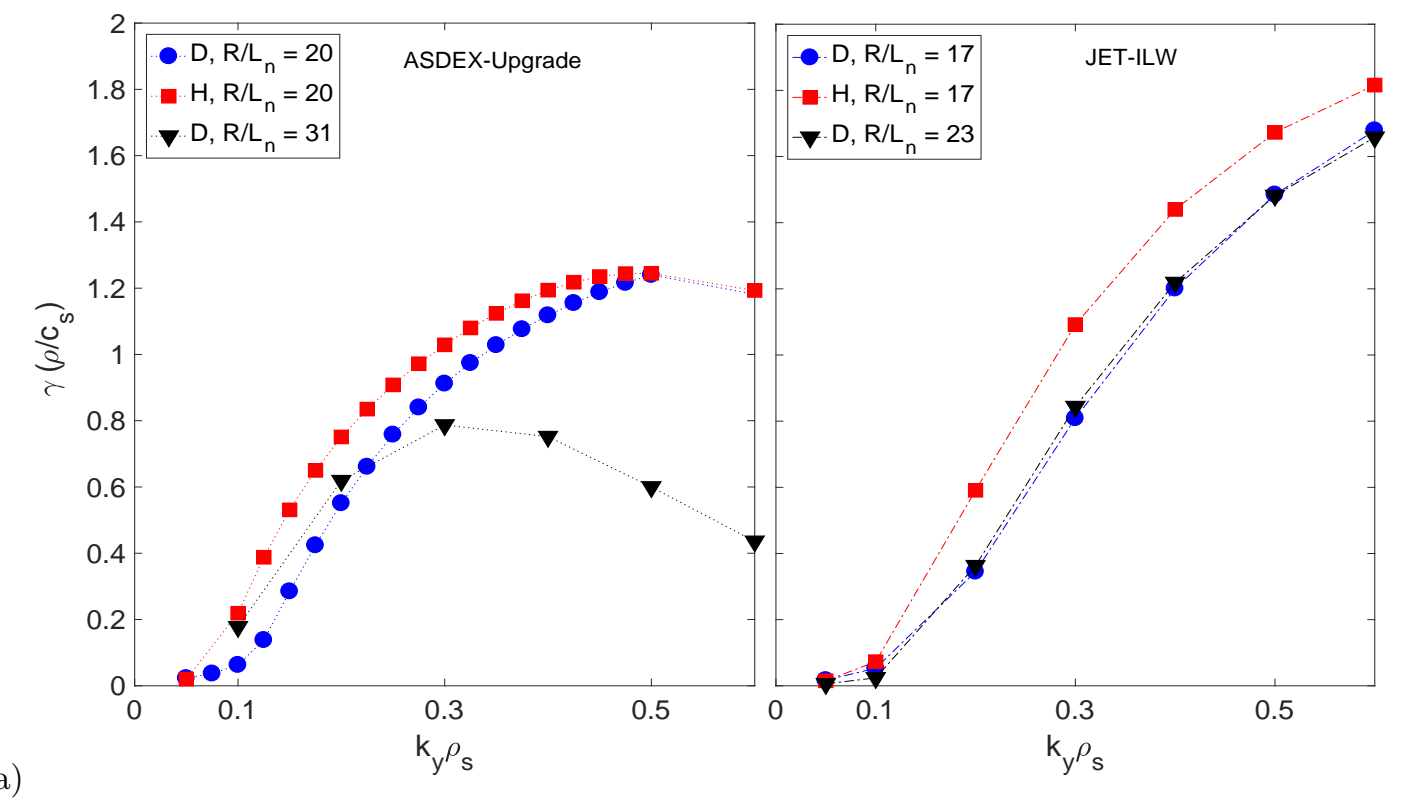

a)
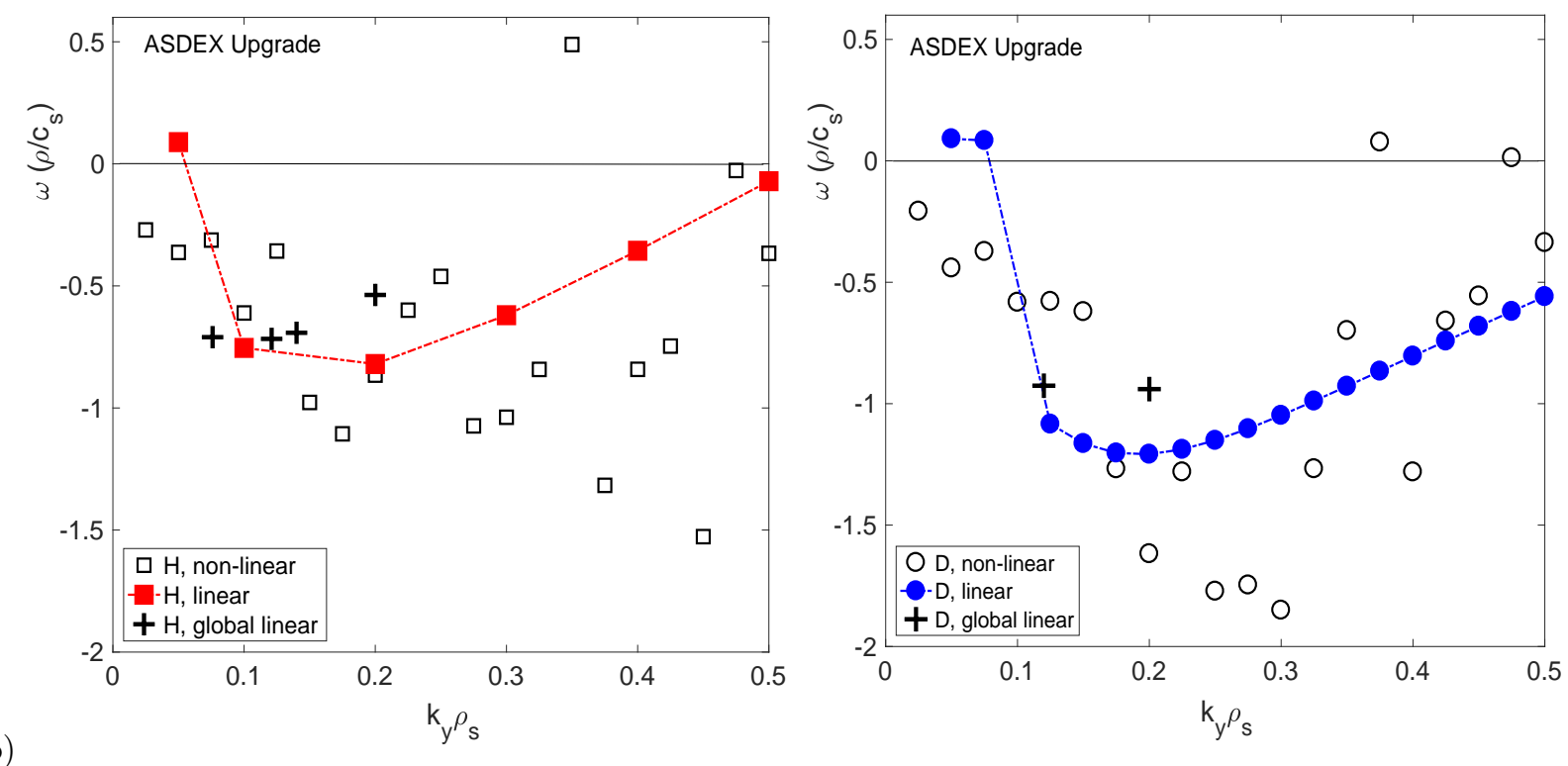

Figure 4: a) Normalized linear growth rate $\gamma\left(\rho / c_{s}\right) \mathrm{v} / \mathrm{s} k_{y} \rho_{s}$ in $H$ and $D$ simulations for JET (right) and ASDEXUpgrade (left). b) Normalized frequency $\omega\left(\rho / c_{s}\right)$ of the modes shown in (a) for ASDEX Upgrade $H$ (left) and D (right) simulations: comparison between linear simulations (full symbols), non-linear simulations (open symbols) and global linear simulations (crosses). 


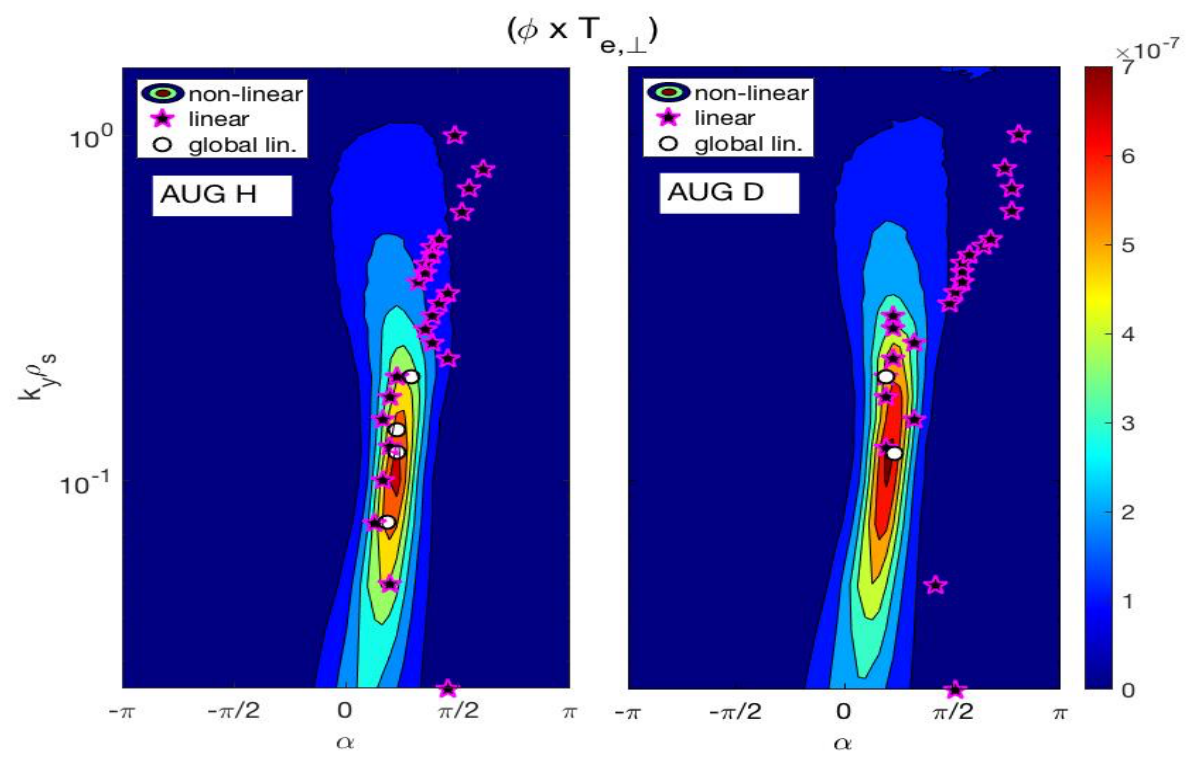

Figure 5: Cross-phase angle $\alpha$ between the electrostatic potential $\phi$ and the electron perpendicular temperature fluctuations $\tilde{T}_{\perp} \mathrm{V} / \mathrm{s} k_{y} \rho_{s}$. Comparison between linear simulations (purple stars), global linear simulations (white circles) and from non-linear simulations for the $R / L_{T e}=39, \beta_{e}=1.8 \cdot 10^{-4}$ case. The non-linear values are amplitude weighted, i.e. the maxim values of the nonlinear cross-phases correspond to the peak in the fluxes $k_{y} \rho_{s}$ spectra.
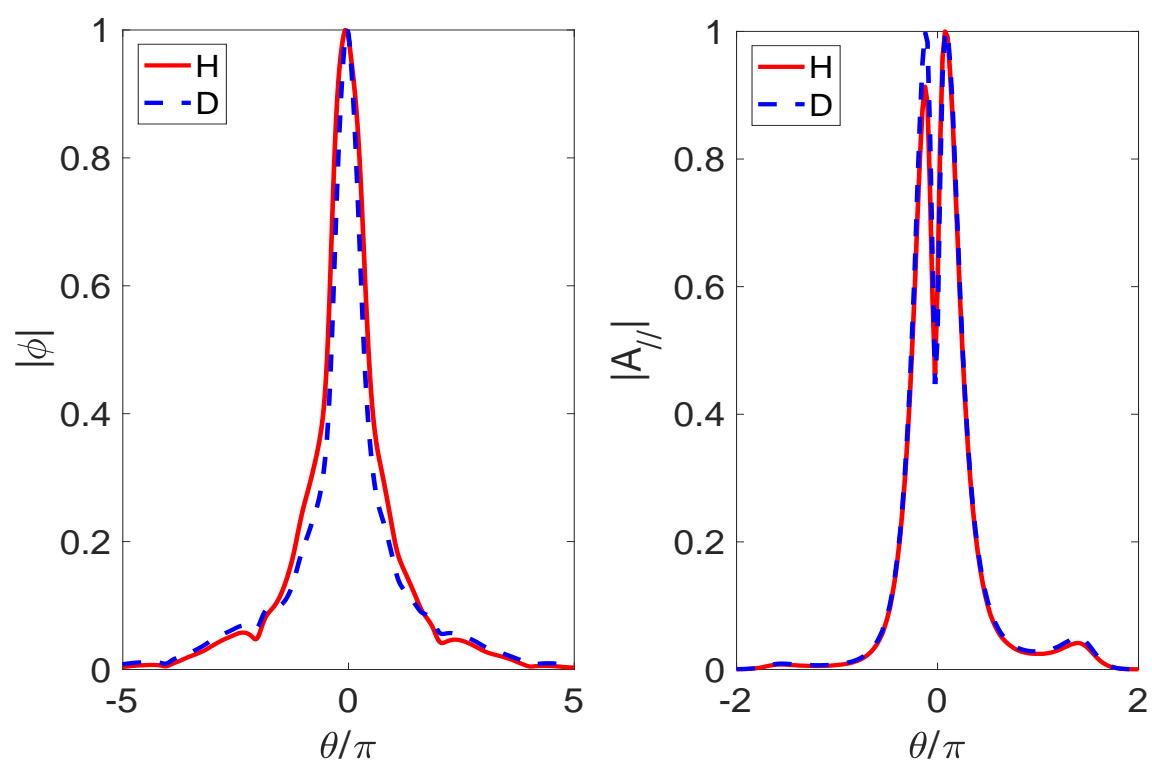

Figure 6: Ballooning representation of $|\phi|$ and $\left|A_{\|}\right|$structure as a function of $\theta / \pi, \theta$ being the poloidal angle along the magnetic field line, from the ASDEX Upgrade linear simulations at $k_{y} \rho_{s}=0.2$. 

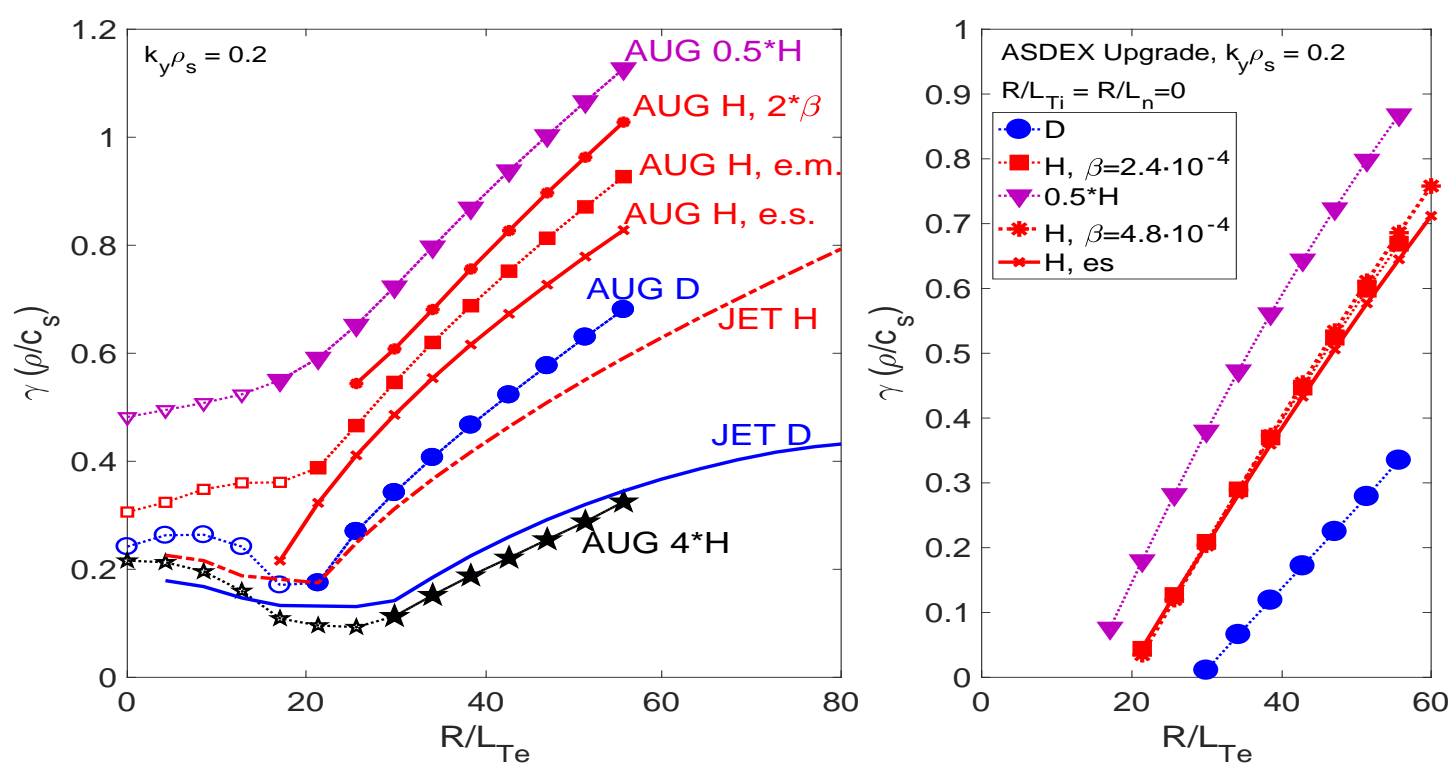

a)
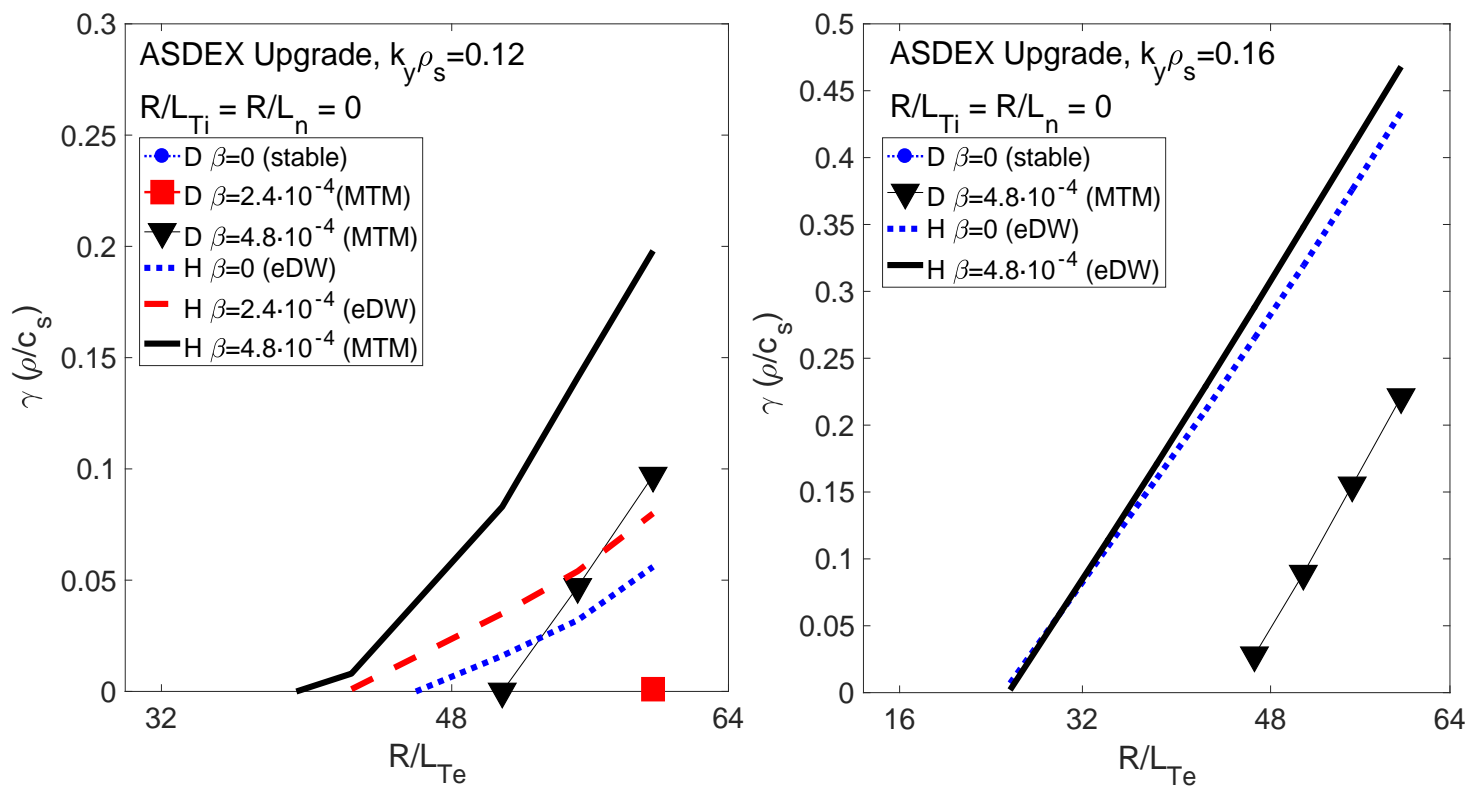

Figure 7: a)Linear growth rate as a function of $R / L_{T e}$ at $k_{y} \rho_{s}=0.2$. The same scan is also repeated for $R / L_{n}=$ $R / L_{T i}=0$ (right). A critical gradient threshold dependent on the ion mass is visible in $R / L_{T e}$. The effect of $\beta_{e}$ has also been studied showing a weak destabilizing effect at $k_{y} \rho_{s}=0.2$. b) Scans of $\gamma\left(\rho / c_{s}\right)$ vs $R / L_{T e}$ using $R / L_{T i}=R / L_{n}=0$ to isolate the effect of $\beta_{e}$ on the linear threshold at $k_{y} \rho_{s}=0.12$ (left) and at $k_{y} \rho_{s}=0.16$ (right). The effect of $\beta_{e}$ is stronger at lower $k_{y}$ and a competition between MTM and drift-wave instabilities appears. 

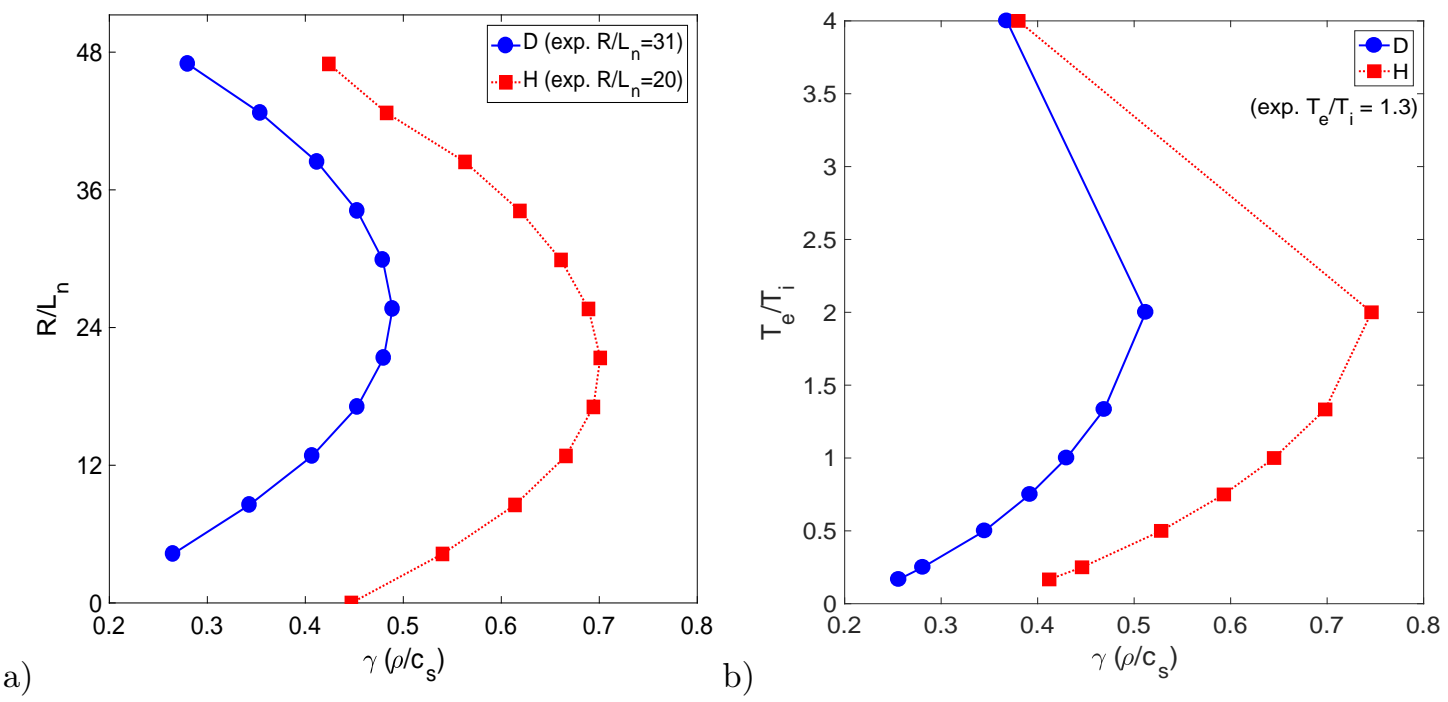

Figure 8: Scans in $R / L_{n}$ (a) and $T_{e} / T_{i}$ (b) of the linear growth rate at $k_{y} \rho_{s}=0.2$. For both $H$ and $D \gamma$ is found to have a non-monotonic behavior with both $R / L_{n}$ and $T_{e} / T_{i}$.
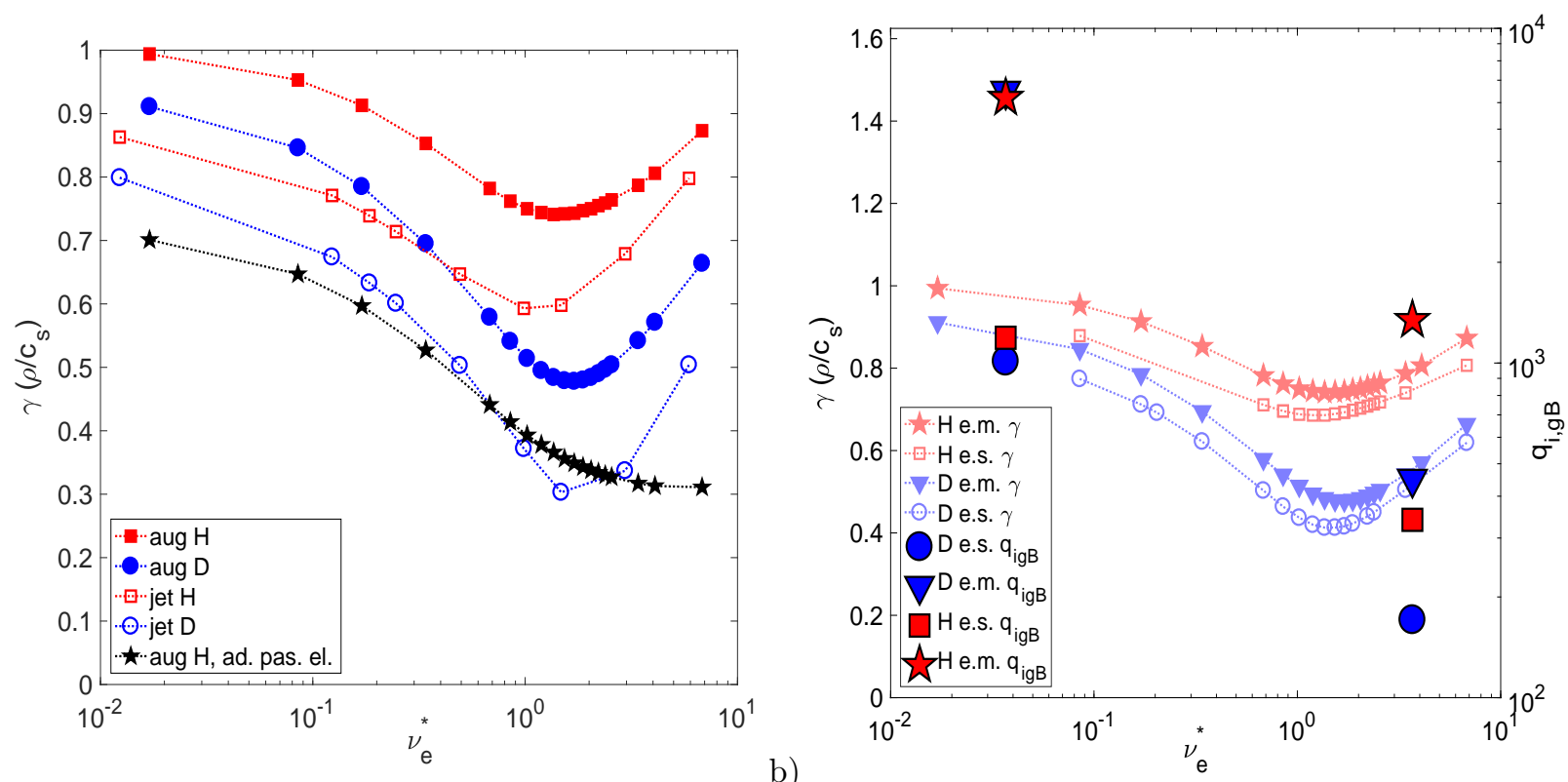

Figure 9: a)Linear growth rate as a function of the collisionality $\nu_{e}^{*}=\nu_{e i} \frac{4}{3 \sqrt{\pi}} \frac{q R^{2}}{\epsilon^{1 \cdot 3}} \frac{1}{v_{t h, e}}$. Comparison between $H$ and $D$ simulations for both JET-ILW and ASDEX Upgrade simulations. As visible in the plot, collisions stabilize up to a certain value of $\nu_{e}^{*}$ corresponding to a minimum in $\gamma$ and destabilize for higher values of collisionality. The difference in the linear growth rate between $H$ and $D$ also depends on $\nu_{e}^{*}$ and shows a maximum difference around the minimum in $\gamma$. When adiabatic passing electrons are considered (black stars), collisions have a stabilizing effect also at high values of $\nu_{e}^{*}$, indicating a dominant role of the passing kinetic electrons dynamics for the linear instability. b) $\gamma\left(\rho / c_{s}\right)$ and $q_{i, g B}$ as a function of the collisionality $\nu_{e}^{*}$ and for different values of $\beta_{e}$ (in the electrostatic case $\beta_{e}=10^{-6}$ while in the electromagnetic case $\left.\beta_{e}=1.8 \cdot 10^{-4}\right)$. Linearly $\beta_{e}$ has a destabilizing effect that does not depend strongly on $\nu_{e}^{*}$. The non-linear flux follows the gyro-Bohm mass scaling at low collisionality while strongly deviates from it at experimental values of $\nu_{e}^{*}$. Furthermore, the electromagnetic effects play a stronger role in the non-linear simulations at both values of $\nu_{e}^{*}$. 

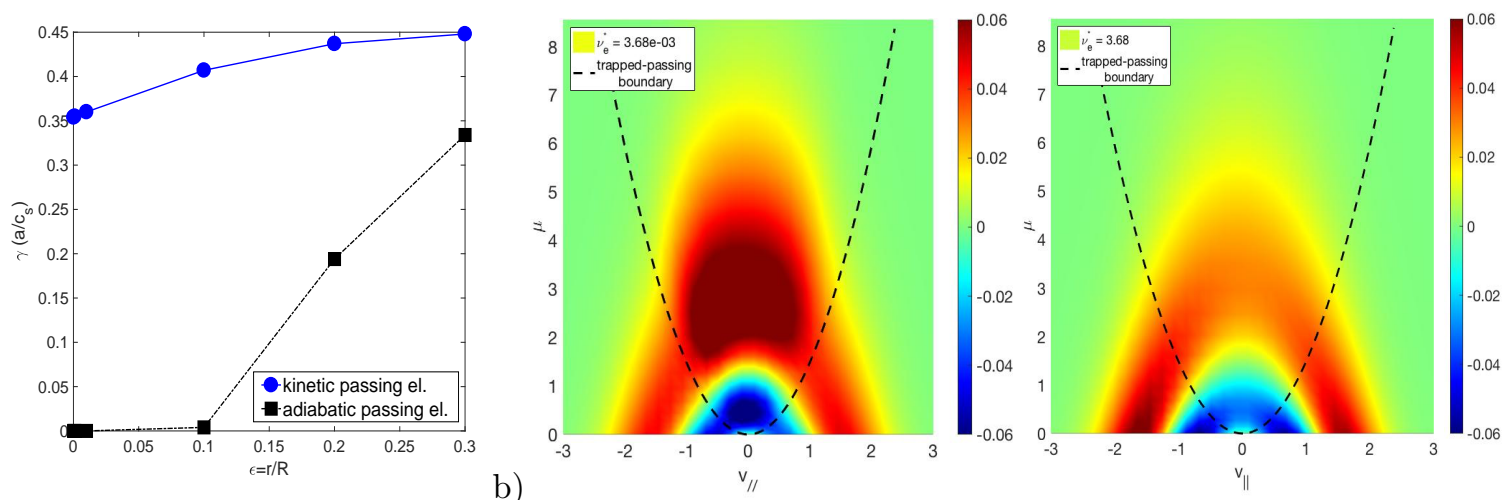

a)

b)

Figure 10: a)Linear growth rate as a function of $\epsilon=r / R$ using an $s-\alpha$ geometry for $\nu_{e}^{*}=3.68$. This corresponds to a scan in the trapped particle fraction. The trapped particles are fundamental with adiabatic passing electrons (as for TEM) but not when kinetic passing electrons are considered. b) Velocity space representation of the electron curvature term, $\gamma_{c u r v}^{e l}\left(v_{\|}, \mu\right)$, contribution to the growth rate. At $\nu_{e}^{*}=0.0368$ (left) the trapped particle region, delimited by the dotted black line, is fundamental. At $\nu_{e}^{*}=3.68$, the passing particle region becomes the dominant region.

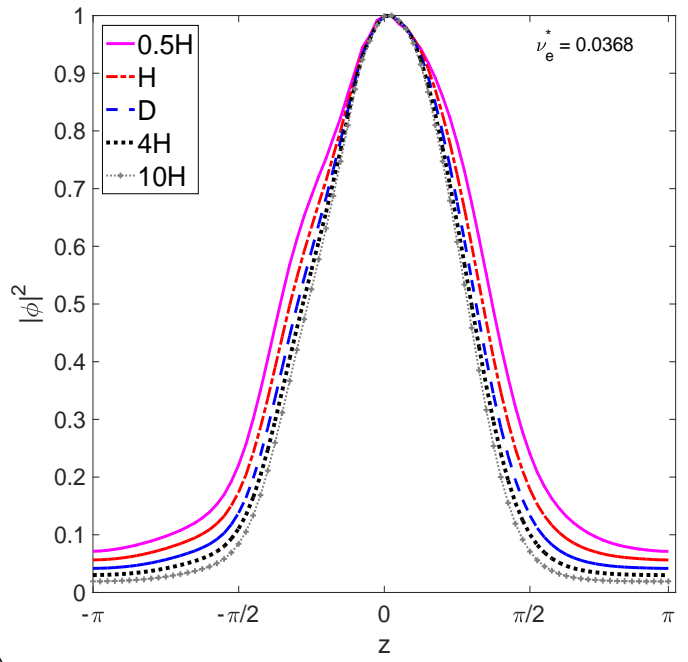

a)

Figure 11: Electrostatic potential parallel structure $\phi(z)$ for different ion mass and for different $\nu_{e}^{*}$ : (a) $\nu_{e}^{*}=0.0368$, b) $\nu_{e}^{*}=3.68$. Larger structures of $\phi(z)$ with lower isotope mass appears at high $\nu_{e}^{*}$, while, at low collisionality, no substantial difference in $\phi(z)$ is observed with different isotope mass. 

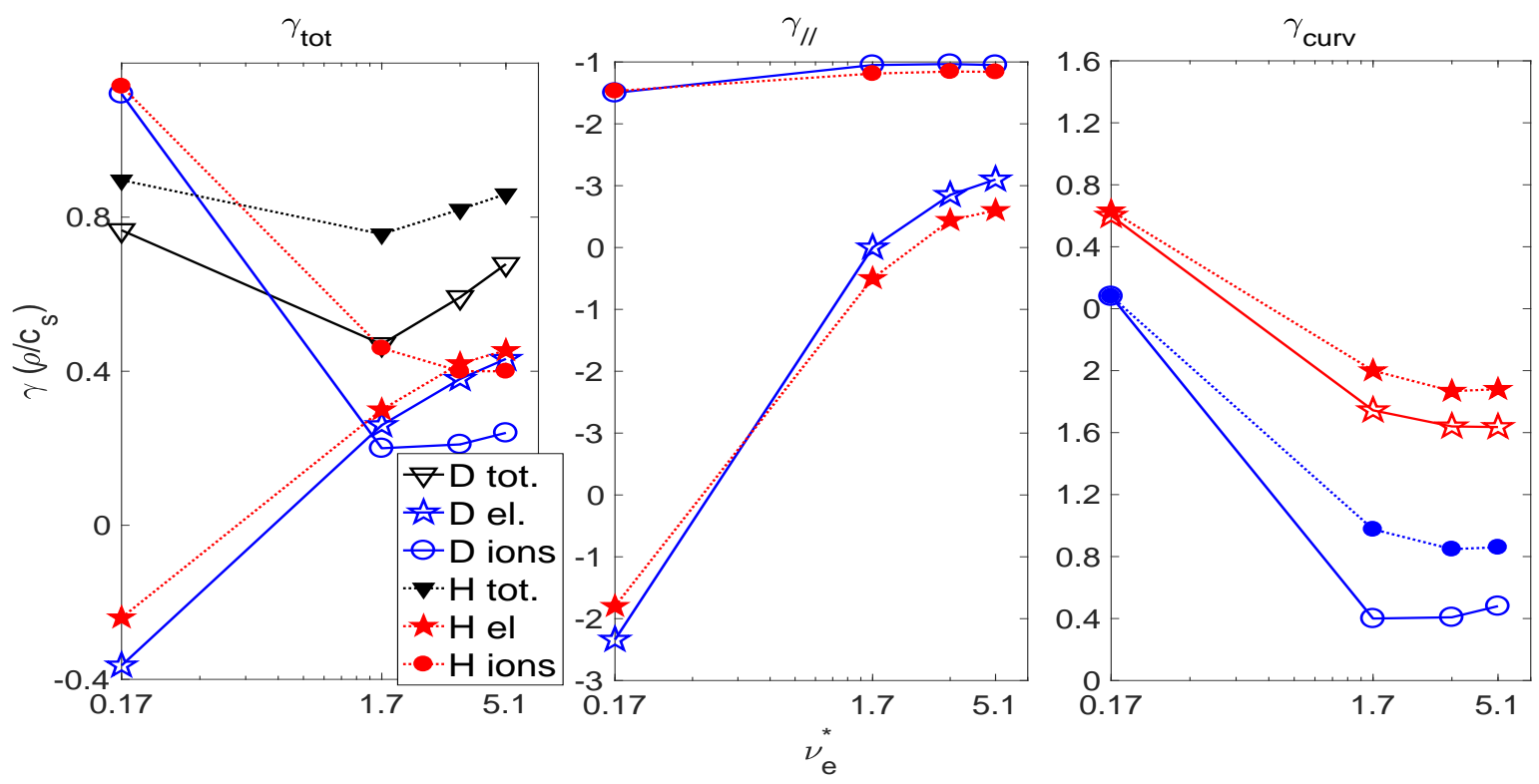

a)
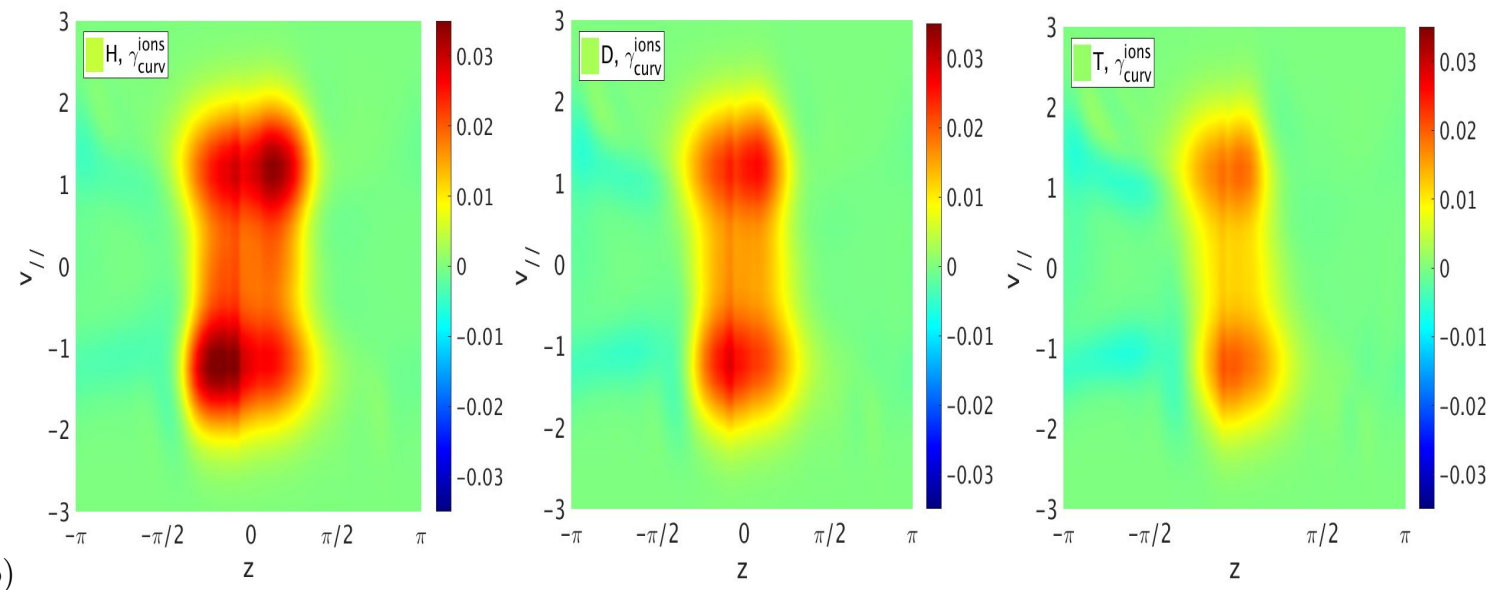

Figure 12: Electrons (stars) and ions (circles) curvature $\left(\gamma_{\text {curv }}\right)$ and parallel $\left(\gamma_{\|}\right)$contributions to the linear growth rate $\gamma_{\text {tot }}$ as a function of $\nu_{e}^{*}$ in $H$ (red) and $D$ (blue) simulations. High collisionality strongly damps the parallel electron stabilizing contributions and enhances the difference between $H$ and $D$ growth rates, especially for the ion $\gamma_{c u r v}$ term. b) Ion curvature term $\gamma_{\text {curv }}^{\text {ions }}$ contribution to the growth rate plotted in the space $\left(z, v_{\|}\right)$at $\nu_{e}^{*}=3.68$ for $H$ (left), D (center) and $T$ (right) isotopes. The larger structures of $\phi(z)$ with lower isotope mass observed at high collisionality influence the contribution of the curvature term to the growth rate of both species and in particular that of the ions. This determines the difference in the ion $\gamma_{\text {curv }}$ between $H$ and $D$ visible in Fig.12a. 

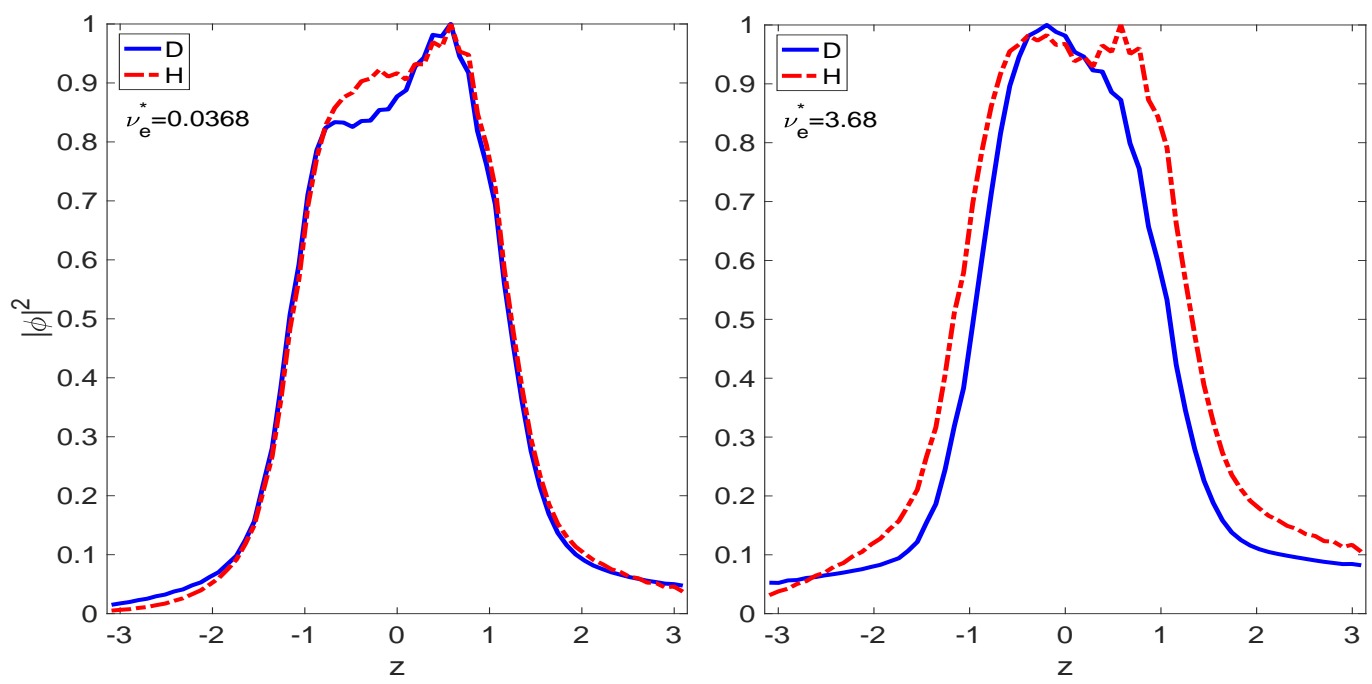

Figure 13: $|\phi(z)|^{2}$ from the ASDEX Upgrade electrostatic nonlinear simulations at $\nu_{e}^{*}=0.0368$ (left) and at $\nu_{e}^{*}=0.0368$ (right). As in the linear simulations, at high collisionality the structures of $\phi$ are broader with lower isotope mass. At low collisionality no substantial difference is observed.

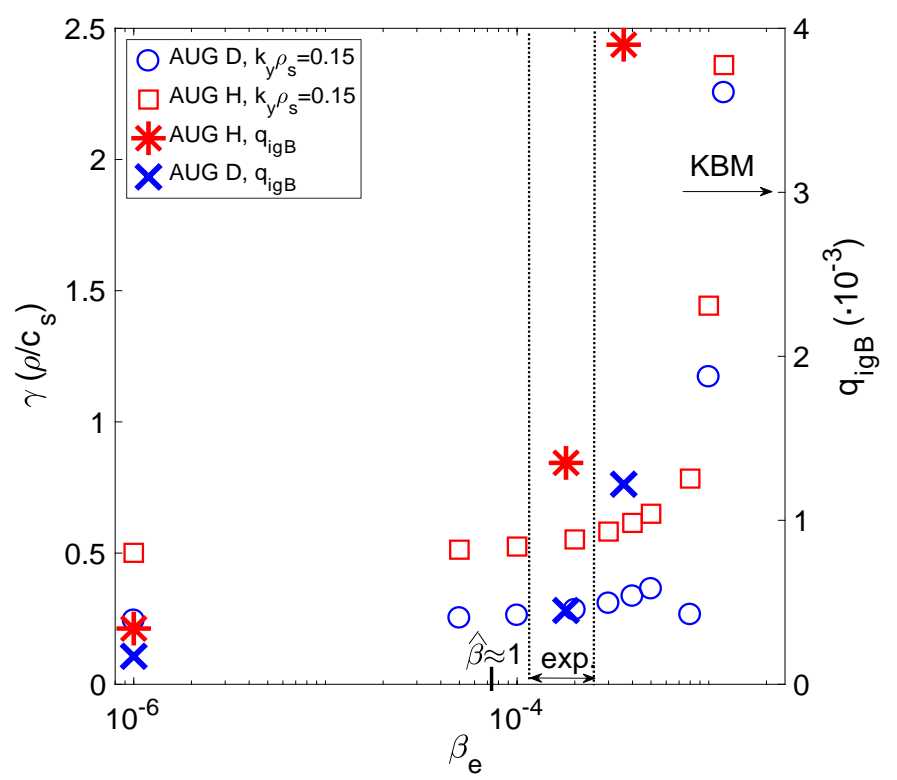

Figure 14: Effect of $\beta_{e}=8 \pi n_{e} T_{e} / B^{2}$ on the linear growth rate at $k_{y} \rho_{s}=0.15$ (similar linear results are obtained in the range $0.05 \leq k_{y} \leq 0.4$ ) and on the ASDEX Upgrade non-linear ion heat flux. Linearly $\beta_{e}$ has a destabilizing effect even if not strong until the KBM limit. Non-linearly a strong destabilization by $\beta_{e}$ is observed at values much lower compared to the linear KBM limit. For a comparison with the results in Ref.[24, 30], where was found that above values of $\hat{\beta}=\beta_{e}\left(\hat{q} R / L_{\perp}\right)^{2} \approx 1$ electromagnetic effect are fundamental in nonlinear simulations, in the ASDEX Upgrade case, $\beta_{e}=1.8 e-04$ corresponds to $\hat{\beta}=2.1$. 

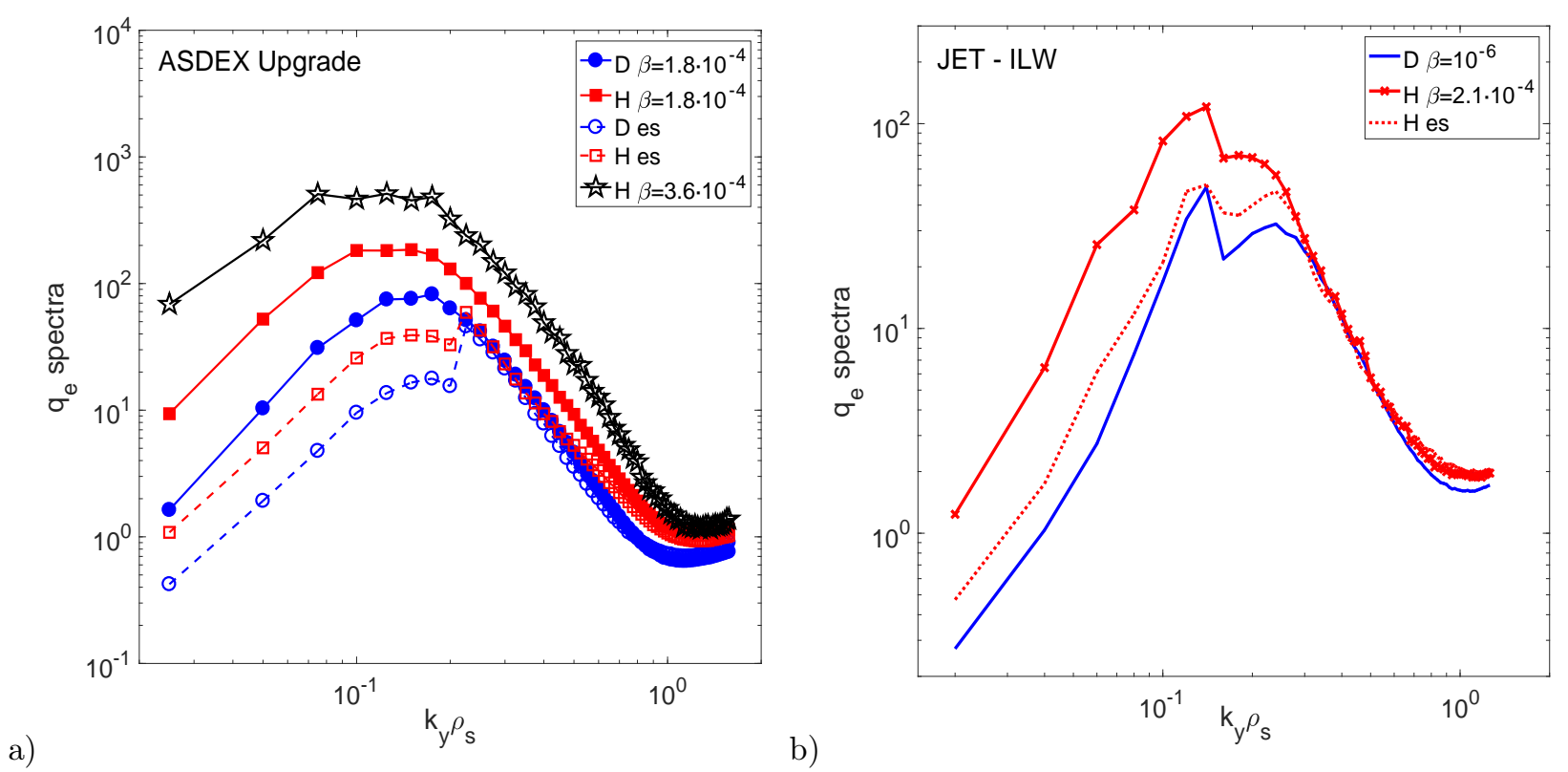

Figure 15: Electron heat flux spectra in $k_{y}$ from the non-linear simulations of ASDEX Upgrade (a) and JET-ILW (b). Increasing $\beta$ leads to an enhancement of the heat fluxes and in particular to a stronger contribution to the fluxes from low $k_{y}$ wave-numbers. When $\beta_{e}$ is further increased, also the peak of the heat flux spectra moves toward lower $k_{y}$ (as visible comparing the red and the black lines in figure a). The same behavior is observed in the $k_{y}$ spectra of $\phi$.
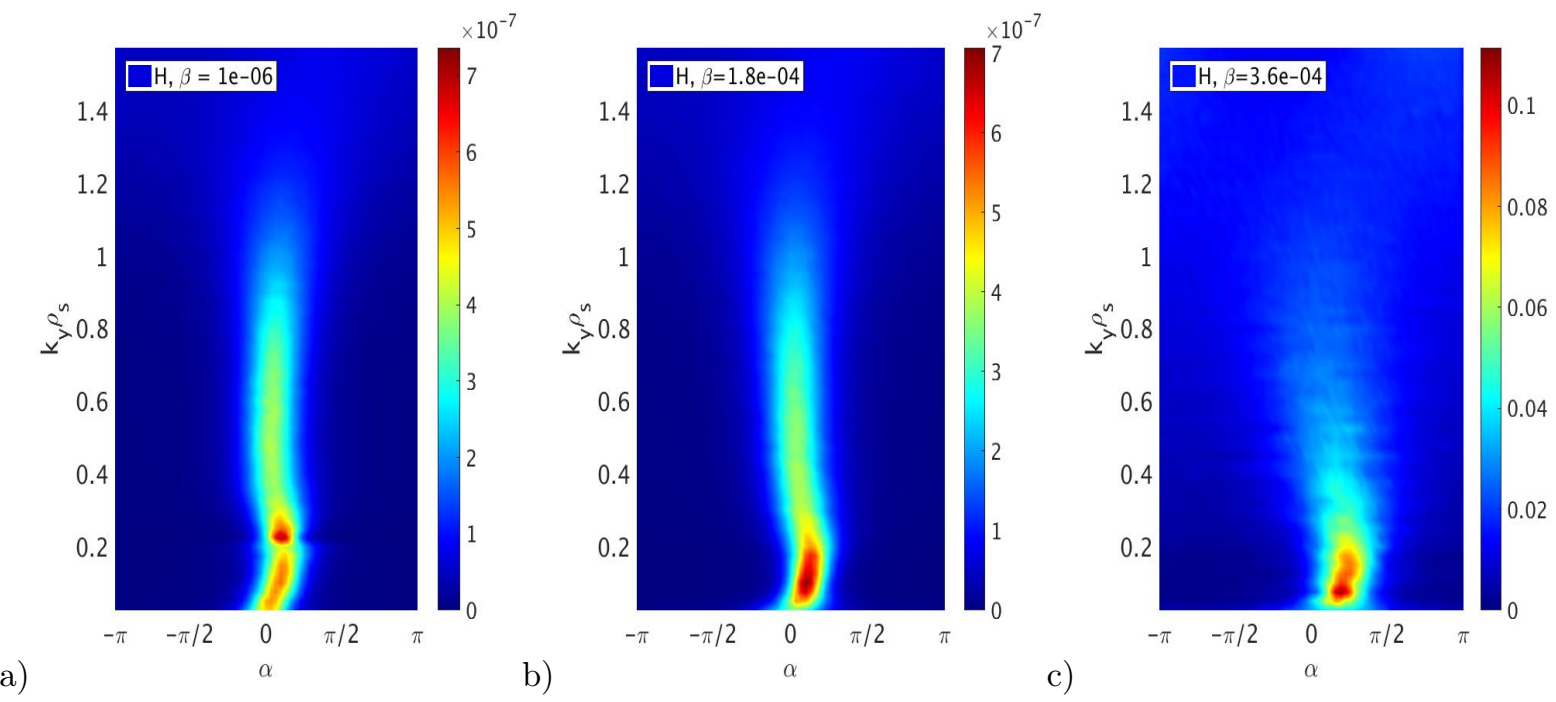

Figure 16: Binormal wave number spectrum of the cross-phase angle $\alpha$ between the electrostatic potential $\phi$ and the perpendicular electron temperature fluctuations $\tilde{T}_{\perp}$ from $H$ non-linear simulations with a) $\left.\beta=10^{-6}, b\right) \beta=1.8 \cdot 10^{-4}$ and c) $\beta=3.6 \cdot 10^{-4}$. Increasing $\beta$ leads to a stronger contribution from low $k_{y}$ wave-numbers and to cross-phases closer to $\pi / 2$ : for $\beta_{e}=10^{-6}$ the cross-phase peaks around $\alpha=\pi / 10$ and $k_{y} \rho_{s}=0.2$; for $\beta_{e}=1.8 \cdot 10^{-4}$ the cross-phase peaks around $\alpha=\pi / 10$ and $k_{y} \rho_{s}=0.1$; for $\beta_{e}=3 \cdot 6 \cdot 10^{-4}$ the cross-phase peaks around $\alpha=\pi / 5$ and $k_{y} \rho_{s}=0.075$. These results support what found in Ref.[24] suggesting that, in non-linear simulations in edge conditions, increasing $\beta$ leads to MHD-like instabilities at values much lower than the ones expected from linear simulations. 

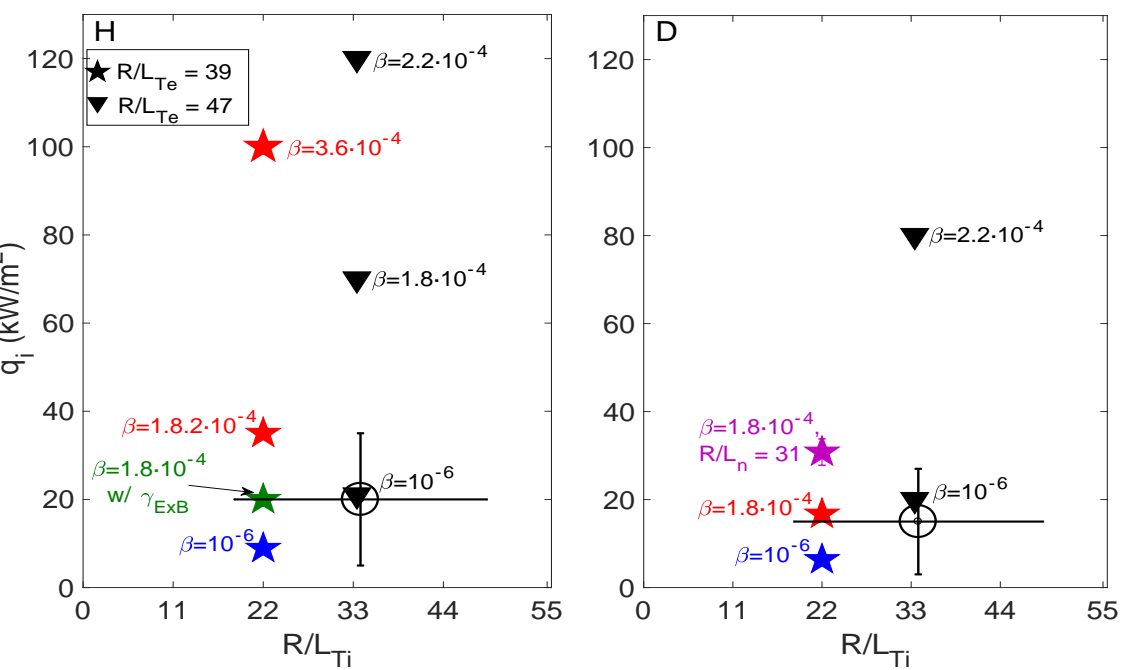

a)
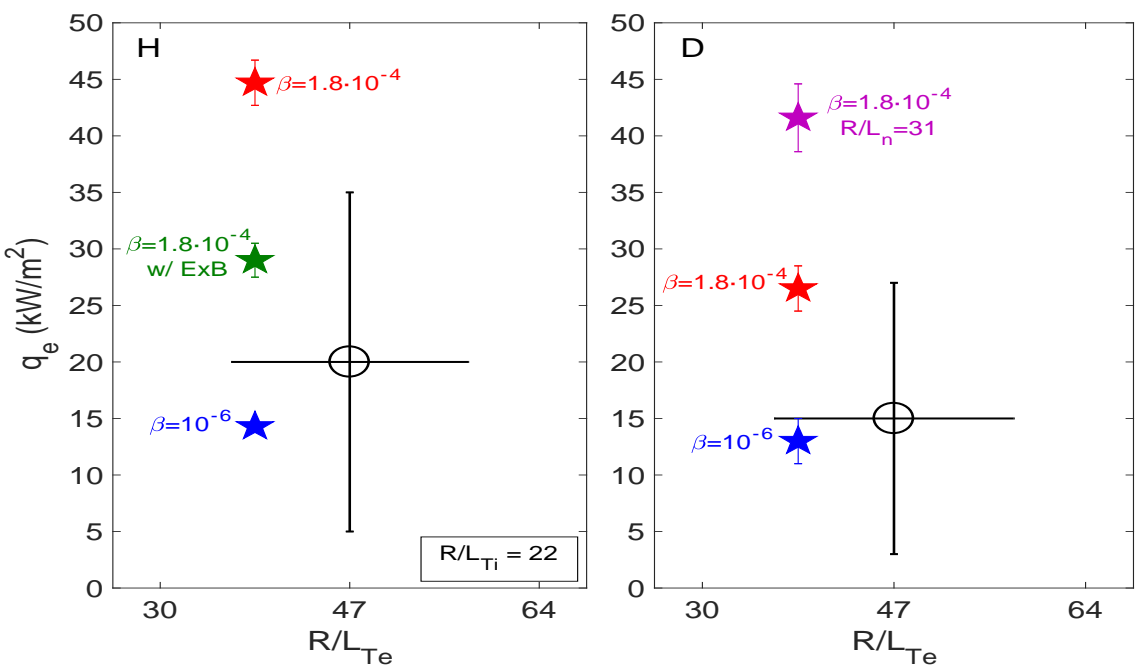

b)
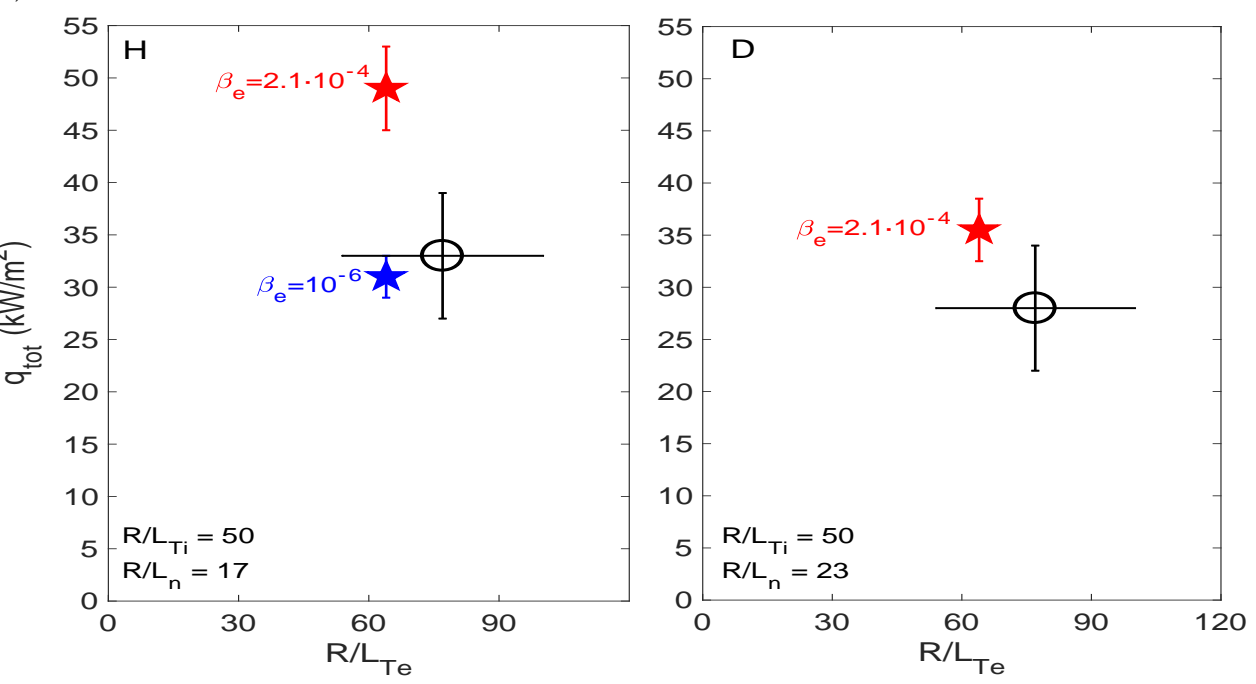

Figure 17: a)Comparison between the experimental heat fluxes in $W / m^{2}$ and the GENE simulations for: a)ASDEX Upgrade, ions; b)ASDEX Upgrade, electrons; c)JET-ILW, total heat flux. The heat fluxes from the electrostatic ( $\beta_{e}=$ $10^{-6}$ ) simulations all match the experimental values within error-bars while, when electromagnetic effect are retained in the simulations, a strong increase of the fluxes is observed and a reduction of $\beta_{e}$ and/or $R / L_{T e}$ is required in order to match the experimental values. For ASDEX Upgrade, $R / L_{n}=20$ is used in the simulations. The effects of the higher value of $R / L_{n}$ in $D\left(R / L_{n}=31\right.$, magenta star and shown in the right plots of figures a and b) and of $\gamma_{E x B}$ (green star and shown in the left plots of figures a and $b$ ) are also tested. Regarding the JET simulations, we point out that in these cases $q_{e} \approx 1.4 q_{i}$. 


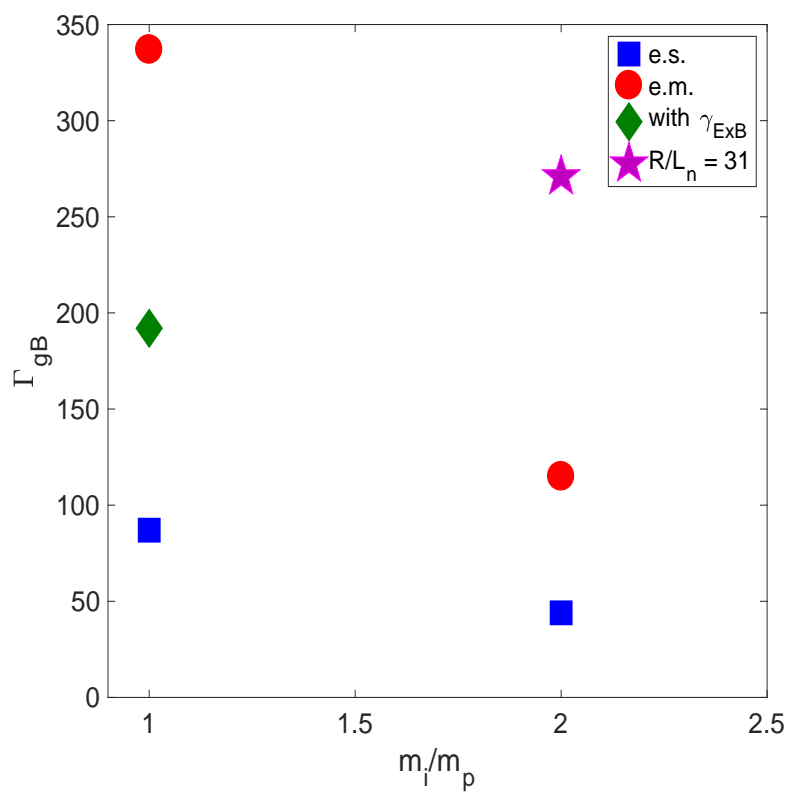

Figure 18: Particle fluxes in gyro-Bohm units as a function of the isotope mass divided by the proton mass from the GENE simulations for ASDEX Upgrade (similar trends are found for JET). In the simulations $R / L_{n}=20$ is used except for one point in $D$ (magenta star) with $R / L_{n}=31$, i.e. the experimental value of $R / L_{n}$ in $D$. The flux from the simulation with the imposed $E \times B$ shear is represented by the green diamond. 